The Astrophysical Journal, 683:304-320, 2008 August 10

(C) 2008. The American Astronomical Society. All rights reserved. Printed in U.S.A.

\title{
PROPLYDS AND MASSIVE DISKS IN THE ORION NEBULA CLUSTER IMAGED WITH CARMA AND SMA
}

\author{
J. A. Eisner ${ }^{1,2}$ and R. L. Plambeck \\ Department of Astronomy, 601 Campbell Hall, University of California, Berkeley, CA 94720; jae@astro.berkeley.edu, \\ plambeck@astro.berkeley.edu \\ John M. Carpenter and S. A. Corder \\ Department of Astronomy MC 105-24, California Institute of Technology, Pasadena, CA 91125; \\ jmc@astro.caltech.edu, sac@astro.caltech.edu \\ AND \\ C. QI AND D. WILNER \\ Harvard-Smithsonian Center for Astrophysics, 60 Garden Street, Mail Stop 42, Cambridge, MA 02138; \\ cqi@cfa.harvard.edu, dwilner@cfa.harvard.edu \\ Received 2008 February 15; accepted 2008 March 19
}

\begin{abstract}
We imaged a $2^{\prime} \times 2^{\prime}$ region of the Orion Nebula Cluster $(\mathrm{ONC})$ in $1.3 \mathrm{~mm}$ wavelength continuum emission with the recently commissioned Combined Array for Research in Millimeter Astronomy (CARMA) and with the Submillimeter Array (SMA). Our mosaics include $>250$ known near-IR cluster members, of which 36 are so-called "proplyds" that have been imaged previously with the Hubble Space Telescope. We detected 40 sources in $1 \mathrm{~mm}$ continuum emission (one of which is the BN Object), and several of them are spatially resolved with our observations. The emission from most objects arises predominantly from dust, and circumstellar masses inferred for detected sources range from 0.01 to $0.5 M_{\odot}$. The average circumstellar mass for undetected sources is estimated to be $\sim 0.001 M_{\odot}$, approximately an order of magnitude smaller than the minimum-mass solar nebula. Most stars in the ONC thus do not appear to currently possess sufficient mass in small dust grains to form Jupiter-mass (or larger) planets. Comparison with previous results for younger and older regions indicates that massive disks evolve significantly on $\sim$ Myr timescales. We also show that the percentage of stars in Orion surrounded by disks more massive than $\sim 0.01 M_{\odot}$ is substantially lower than in Taurus, indicating that environment has an impact on the disk-mass distribution. Disks in Orion may be truncated through photoevaporation caused by the intense radiation field of the Trapezium stars, and we see marginal evidence for such a scenario in the spatial distribution of massive disks within the cluster. Our data show no statistically significant correlation between disk and stellar masses, although we see hints of a higher percentage of massive disks around lower mass stars.
\end{abstract}

Subject headings: open clusters and associations: individual (Orion) — planetary systems: protoplanetary disks stars: pre-main-sequence

\section{INTRODUCTION}

The existence of protoplanetary disks around young stars is now firmly established. High-resolution images from optical to radio wavelengths have shown disklike morphologies and Keplerian rotation profiles around a number of young stars (e.g., O'Dell \& Wong 1996; McCaughrean \& O’Dell 1996; Padgett et al. 1999; Eisner et al. 2004; Koerner \& Sargent 1995; Dutrey et al. 1996; Wilner et al. 2000). Moreover, observations of near-IR emission from young stars in excess of that expected from their stellar photospheres imply that most stars aged less than a few million years possess inner circumstellar disks (e.g., Strom et al. 1989; Haisch et al. 2001).

Protoplanetary disks are the birth sites of planetary systems, and the ubiquity, properties, and lifetimes of disks constrain the timescales and mechanisms of planet formation. The mass distribution of protoplanetary disks is especially important, since disk mass is related to the mass of the planets that may potentially form. For our own solar system, the masses of planets and other bodies can be used to reconstruct a minimum-mass solar nebula (MMSN)

\footnotetext{
${ }^{1}$ Miller Institute for Basic Research in Science, Berkeley, CA 94720.

2 Steward Observatory, University of Arizona, Tucson, AZ 85721.
}

describing the amount of solar composition material needed to build the solar system. Depending primarily on the core masses (and hence chemical compositions) of Jupiter and Saturn (which are not known precisely), estimates of the MMSN range from $\sim 0.01$ to $0.1 M_{\odot}$ (Weidenschilling 1977). Such disk masses are also required by planet formation models to build giant planets on timescales shorter than inferred disk lifetimes (e.g., Hayashi 1981; Alibert et al. 2005). The MMSN is thus an informative benchmark against which to gauge the potential of disks around other stars to form solar systems like our own.

A widely used method (e.g., Beckwith et al. 1990) for measuring disk masses is to observe emission from optically thin dust, and then use assumed dust grain properties to convert observed fluxes into dust masses. An assumed gas-to-dust ratio is then used to estimate the total (gas + dust) circumstellar mass. At short wavelengths $(\lambda \lesssim 10 \mu \mathrm{m})$, the dust in protoplanetary disks is optically thick, even for masses $<10^{-6} M_{\odot}$. Observations at submillimeter and millimeter wavelengths are necessary to measure optically thin dust emission, and hence to determine the total mass of dust in the disk.

Several investigators have carried out comprehensive single-dish millimeter and submillimeter continuum surveys toward regions of star formation that comprise loose aggregates of stars: Taurus 
(Beckwith et al. 1990; Osterloh \& Beckwith 1995; Motte \& André 2001; Andrews \& Williams 2005), $\rho$ Ophiuchi (André \& Montmerle 1994; Nuernberger et al. 1998; Motte et al. 1998; Andrews \& Williams 2007), Lupus (Nuernberger et al. 1997), Chamaeleon I (Henning et al. 1993), Serpens (Testi \& Sargent 1998), and MBM 12 (Itoh et al. 2003; Hogerheijde et al. 2002). In Taurus, $22 \%$ of stars surveyed appear to possess disks more massive than $\sim 0.01 M_{\odot}$, and the median disk mass is $5 \times 10^{-3} M_{\odot}$ (Andrews \& Williams 2005). The fraction of massive disks ${ }^{3}$ and the median disk mass are comparable in $\rho$ Ophiuchi (André \& Montmerle 1994; Andrews \& Williams 2007).

However, low-density star-forming regions are not the typical birth sites of stars; rather, most stars form in rich clusters like the Orion Nebula (Lada et al. 1991, 1993; Carpenter 2000; Lada \& Lada 2003). Isotopic abundances in our solar system suggest that it too may have formed in a dense, Orion-like environment (e.g., Hester \& Desch 2005; Williams \& Gaidos 2007). Expanding millimeter continuum surveys to include rich clusters allows the determination of the frequency and evolution of massive disks in typical star (and planet) formation environments. The high stellar density in rich clusters also allows the assembly of good statistics, since many disks can be mapped at once.

The main challenge to observing rich clusters at (sub)millimeter wavelengths is that very high angular resolution is required to resolve individual sources and to distinguish compact disk emission from the more extended emission of the molecular clouds in which young clusters are typically embedded. Single-aperture millimeter-wavelength telescopes lack sufficient angular resolution, and to date only three rich clusters have been observed with millimeter-wavelength interferometers: the Orion Nebula cluster (Mundy et al. 1995; Bally et al. 1998b; Williams et al. 2005; Eisner \& Carpenter 2006), IC 348 (Carpenter 2002), and NGC 2024 (Eisner \& Carpenter 2003).

These interferometric surveys of rich clusters have detected very few disks with $\gtrsim 0.01-0.1 M_{\odot}$ of material, in large part because of limited sensitivity and areal coverage. The most recent observations of Orion detected emission from several massive $\left(\gtrsim 0.01 M_{\odot}\right)$ disks (Williams et al. 2005; Eisner \& Carpenter 2006), while upper limits from other surveys range from $\sim 0.025$ to $0.17 M_{\odot}$ (Mundy et al. 1995; Bally et al. 1998b). Considering as ensembles the large numbers $(\gtrsim 100)$ of young stars included in the cluster surveys allowed estimates of mean disk masses of $\sim 0.002,0.005$, and $0.005 M_{\odot}$ in IC 348, NGC 2024, and the Orion Nebula Cluster (ONC), respectively (Carpenter 2000; Eisner \& Carpenter 2003, 2006). Thus, it appears that many stars aged $\lesssim 1$ Myr still possess massive circumstellar disks, although more sensitive observations are necessary to directly detect large numbers of massive disks at a range of ages, and thereby constrain the mass distribution and evolutionary timescales.

Here, we present a new $1.3 \mathrm{~mm}$ wavelength interferometric survey of the ONC, a young, embedded stellar cluster that includes the bright, massive Trapezium stars. Our observations make use of the Submillimeter Array (SMA) and the recently commissioned Combined Array for Research in Millimeter Astronomy (CARMA). The combination of data from these two instruments yields a map of the millimeter-wavelength continuum emission in Orion with unprecedented sensitivity, angular resolution, and image fidelity.

The Trapezium region contains hundreds of stars within a several arcminute radius, and pre-main-sequence evolutionary models

\footnotetext{
3 Here and throughout the text, "massive disks" refer to disks with mass comparable to or greater than $0.01 M_{\odot}$, the lower range of estimates for the minimummass solar nebula.
}

(e.g., D’Antona \& Mazzitelli 1994) fitted to spectroscopic and/or photometric data indicate that most stars are less than approximately 1 million years old (e.g., Prosser et al. 1994; Hillenbrand 1997). The standard deviation in the distribution of inferred stellar ages is $\lesssim 1$ Myr (Hillenbrand 1997). Our observations thus provide a snapshot of millimeter emission around a large number of roughly coeval young stars.

With the large number of stars in the ONC, we can also investigate the correlation of disk properties with stellar and/or environmental properties. Previous investigations of near-IR excess emission showed the inner disk fraction for stars in Orion to be largely independent of stellar age and mass, although there are indications of a paucity of disks around very massive stars (Hillenbrand et al. 1998; Lada et al. 2000). In addition, the inner disk fraction may decrease at larger cluster radii (Hillenbrand et al. 1998). The millimeter observations presented here enable investigation of how such stellar and environmental properties correlate with disk mass.

We adopt a distance to the ONC of $400 \mathrm{pc}$, based on recent trigonometric parallax measurements of several stars (Sandstrom et al. 2007; Menten et al. 2007) and orbital fitting for a spectroscopic binary (Kraus et al. 2007). This is substantially lower than the value of $480 \mathrm{pc}$ computed based on statistical parallax of water maser spots (Genzel et al. 1981), which was adopted in previous studies of the ONC (e.g., Eisner \& Carpenter 2006). We discuss below the importance of this revised distance to our results.

\section{OBSERVATIONS AND DATA REDUCTION}

\subsection{CARMA Observations and Calibration}

We mosaicked a $2^{\prime} \times 2^{\prime}$ region toward the $\mathrm{ONC}$ in $\lambda 1.3 \mathrm{~mm}$ continuum with CARMA between 2007 October and December. CARMA consists of six $10 \mathrm{~m}$ antennas and nine $6 \mathrm{~m}$ antennas situated at $2200 \mathrm{~m}$ elevation at Cedar Flat in the Inyo Mountains of California. With a total of 15 antennas, CARMA provides 105 baselines, enabling excellent coverage of the $u-v$ plane, and hence high image fidelity. Two different array configurations (" $\mathrm{C}$ " and " $\mathrm{B}$ ") were used to obtain antenna separations ranging from 30 to $946 \mathrm{~m}$.

Continuum data were recorded in six $\sim 500 \mathrm{MHz}$ bands covering the frequency ranges $221.75-223.25$ and $227.25-228.75 \mathrm{GHz}$ from the receivers' lower and upper sidebands, respectively. Each band consists of 15 channels. Spectral line emission in Orion is mostly resolved out by these observations; that is, across most of the mapped region, it is detected only weakly because it is spatially extended relative to the interferometer fringe spacings. The spectral lines most visible on the shortest baselines, mostly toward the $\mathrm{BN} / \mathrm{KL}$ region in the northwest corner of the mosaic, are the 11(1,11)-10(0,10) transition of $\mathrm{SO}_{2}$ at $221.965 \mathrm{GHz}$ and the $25-24$ transition of $\mathrm{HC}_{3} \mathrm{~N}$ at $227.402 \mathrm{GHz}$. Even these lines are almost completely resolved out for projected antenna spacings $>70 \mathrm{k} \lambda$ (used to generate the final maps; see $\S 2.3$ ). We therefore assume that our bands are effectively line free.

The mosaic consists of 16 pointing centers (Fig. 1), separated by $26^{\prime \prime}$. This separation is comparable to the full-width at halfmaximum (FWHM) beamwidth of the $10 \mathrm{~m}$ antennas, but $\sqrt{3}$ smaller than the FWHM beamwidth of the $6 \mathrm{~m}$ antennas. A twodimensional mosaic is Nyquist sampled if pointings are separated by $\leq \mathrm{FWHM} / \sqrt{3}$. Thus, the CARMA mosaic is Nyquist sampled only for the $6 \mathrm{~m}$ dishes. However, Nyquist sampling is not crucial, since we are interested only in compact sources, rather than extended emission. Simulated CARMA mosaics of a synthetic star field showed that $26^{\prime \prime}$ spacings provided the best balance of 


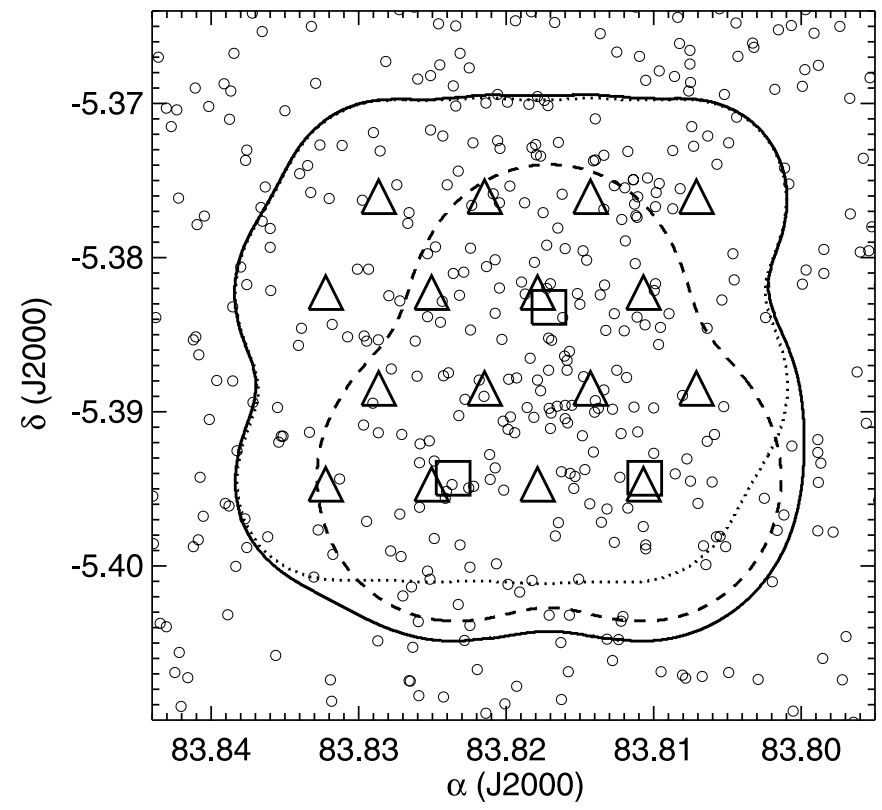

FIG. 1.-Pointing positions for the CARMA mosaic (triangles) and the SMA mosaic (squares) plotted over the positions of $K$-band sources in the ONC (circles). These $K$-band source positions are drawn from Hillenbrand \& Carpenter (2000) and have been registered to the $2 \mathrm{MASS}$ astrometric grid. The unit-gain contours of the CARMA mosaic (dotted curve), the SMA mosaic (dashed curve), and the mosaic produced from the combined SMA+CARMA data set (solid curve) are also indicated.

sensitivity and areal coverage to maximize the number of sources detected.

Each night, we interleaved 16 minute observations of the ONC mosaic (with 1 minute integration time on each pointing center) with 3 minute observations of the phase and amplitude calibrator $\mathrm{J} 0530+135$. Observing the mosaic in its entirety every 20 minutes and repeating this multiple times during the night ensured a highquality synthesized beam and equal sensitivity for each pointing center. The total integration time for the maps was 34 minutes per pointing center.

Telescope pointing was checked and updated on 20 minute intervals using optical counterparts very near to the source. These objects were observed with optical cameras mounted on the dishes, and radio-optical offsets were calibrated periodically. Gain stability, especially in $1 \mathrm{~mm}$ observations like those presented here, is found to be enhanced through this method (S. A. Corder et al., in preparation).

We measured a $1.3 \mathrm{~mm}$ flux density of $3.0 \pm 0.3 \mathrm{Jy}$ for $\mathrm{J} 0530+$ 135 using Uranus as a primary flux calibrator, based on observations during several nights in October when both sources were observed. J0530+135 was also used to calibrate the passband. All calibrations for these data were performed with the MIRIAD package (Sault et al. 1995).

\subsection{SMA Observations and Calibration}

We mosaicked a triangular region approximately $1.7^{\prime}$ on a side and consisting of three pointings with the SMA between 2005 September and 2006 February (Fig. 1). The SMA consists of eight $6 \mathrm{~m}$ dishes near the summit of Mauna Kea in Hawaii. However, for our observations, only seven antennas were available, providing 21 baselines between 10 and $220 \mathrm{~m}$. The $u-v$ coverage for our SMA observations is substantially sparser than for our CARMA observations, and thus the image fidelity is worse (which means, for example, that strong emission is scattered more strongly into other regions of the map). However, the high altitude of the SMA enables very low-opacity observing conditions. The low opacities, combined with increased observing time per pointing, lead to substantially better sensitivity relative to the CARMA observations.

Double-sideband receivers were tuned to a local oscillator (LO) frequency of $225.333 \mathrm{GHz}$. The SMA digital correlator was configured with 24 partially overlapping bands of $104 \mathrm{MHz}$ width in each sideband. Each sideband provided $2 \mathrm{GHz}$ of bandwidth, centered $\pm 5 \mathrm{GHz}$ away from the LO frequency. The double-sideband (DSB) system temperatures were between 80 and $200 \mathrm{~K}$.

As for the CARMA mosaic, we observed the mosaic multiple times throughout the night, obtaining equal integration times for each pointing position. The pointings in the mosaic were separated by $\sim 44^{\prime \prime}$, the approximate FWHM for the SMA dishes at this observing wavelength. As for the CARMA map, the SMA mosaic is larger-than-Nyquist sampled, which provides enhanced areal coverage compared to a Nyquist-sampled mosaic.

We used J0423-013 and 3C 120 as gain calibrators for these tracks, with flux densities of 1.4 and $1.0 \mathrm{Jy}$, respectively, derived using Uranus as a primary flux calibrator. We estimate that the absolute flux scale is uncertain by $\sim 10 \%$. We calibrated the passband using the quasar 3C 454.3 and Uranus where available. Because the $\mathrm{CO}(2-1)$ transitions are present in the observing window, we edited out the parts of the band with strong lines and generated a line-free continuum channel. All calibrations were performed using the SMA adaptation of the IDL-based data reduction package MIR developed at Caltech; calibrated data were then converted into MIRIAD format for further processing.

\subsection{Mapping}

We made mosaics of our CARMA and SMA data sets individually, and after combining the two data sets in the $u-v$ plane. For the individual and combined data sets, we mosaicked the individual pointings into a single image, weighting the data by system temperature and by $u$ - $v$ distance (with a "robust" parameter of 0.5); we then deconvolved and CLEANed them (all using MIRIAD). The angular resolution afforded by the longest baseline data in our maps (from the CARMA B array) is $\sim 0.3^{\prime \prime}$. Our mosaics have $0.1^{\prime \prime}$ pixels, which ensures adequate sampling of individual resolution elements.

Since we are primarily interested in compact disk emission, we eliminated $u-v$ spacings shorter than $70 \mathrm{k} \lambda$ (i.e., with projected baselines shorter than $93 \mathrm{~m}$ ) in order to reduce contamination from bright extended emission. The eliminated spacings correspond to size scales larger than $\sim 3^{\prime \prime}$. The cutoff value was chosen to minimize the rms background noise in the CLEANed images; we measured the rms for $u-v$ cutoff radii of $50,60,70$, and $80 \mathrm{k} \lambda$, and found the $70 \mathrm{k} \lambda$ cutoff to be optimal.

Mosaics produced from our robustly weighted data with $r_{u-v}>$ $70 \mathrm{k} \lambda$ are shown in Figures $2-4$. In the figures, we have divided by the theoretical sensitivity at each location in the image in order to visually downweight the noisier edges of the mosaic (where there are fewer overlapping pointings); we do not divide by the sensitivity in the analysis presented below. We note that even within the uniform (theoretical) sensitivity region of the mosaic, the rms varies substantially because of emission scattered from bright compact and extended sources in the BN/KL and OMC1-S regions located in the upper and lower right quadrants of the maps.

For the SMA mosaic, the unit-gain region (within which the theoretical sensitivity does not vary substantially) encompasses a roughly triangular region covering $\sim 2 \operatorname{arcmin}^{2}$. The rms of 


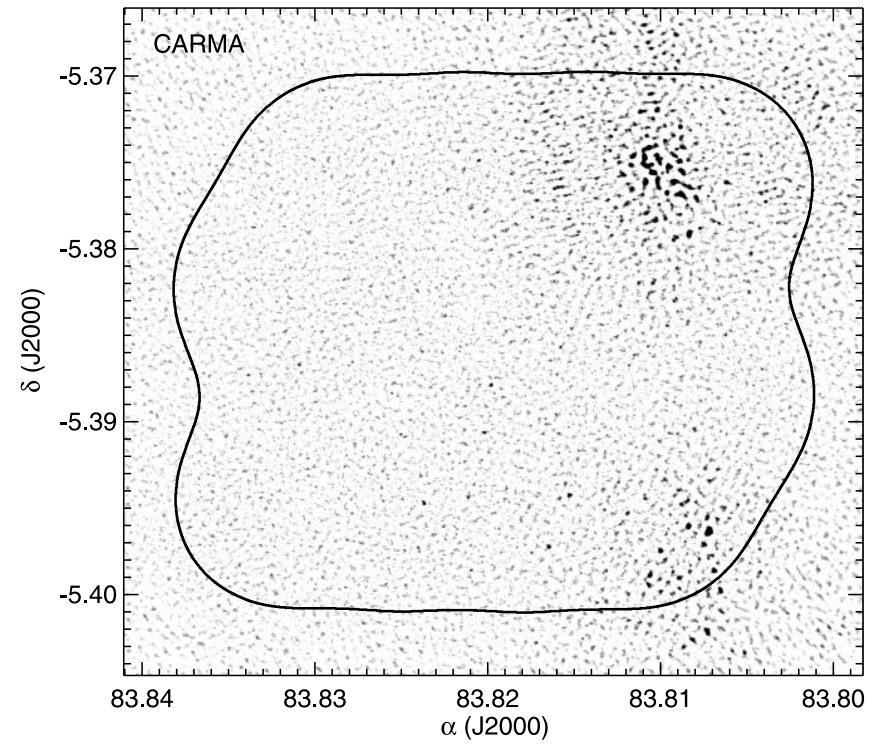

FIG. 2.-ONC imaged in $\lambda 1.3 \mathrm{~mm}$ continuum with CARMA. Only data observed on long baselines $\left(r_{u^{-} v}>70 \mathrm{k} \lambda\right)$ were used to create this image. The unitgain region of the mosaic is indicated by a solid curve. The synthesized beam has a FWHM of $0.61^{\prime \prime} \times 0.52^{\prime \prime}$ at a P.A. of $70^{\circ}$. The rms noise varies within the unit-gain contour from 2.3 to $23 \mathrm{mJy}(1 \sigma)$, with a mean rms of $4.6 \mathrm{mJy}$. Partially resolved extended emission in the $\mathrm{BN} / \mathrm{KL}$ and OMC1-S regions increases the noise level in the upper and lower right corners of the image. The map has been divided by the theoretical rms to visually downweight the noisier edges.

pixels within "clean" regions of the unit-gain contour (i.e., away from the crowded $\mathrm{BN} / \mathrm{KL}$ and $\mathrm{OMC1-S}$ regions) is $0.8 \mathrm{mJy}$. The unit-gain region of the CARMA mosaic encompasses a $2^{\prime} \times$ $2^{\prime}$ area, with an rms noise level (again, in clean regions of the map) of $2.3 \mathrm{mJy}$. For the combined map, which will be used for the bulk of our analysis, the unit-gain region is slightly larger than for the CARMA-only mosaic, and the rms noise level is $1.8 \mathrm{mJy}$. The synthesized beam has dimensions (FWHM) of $0.69^{\prime \prime} \times 0.60^{\prime \prime}$ at a position angle of $72^{\circ}$.

\section{ANALYSIS AND RESULTS}

\subsection{Detection Thresholds}

Because the map contains a large number of pixels, we must employ a fairly high detection threshold to avoid random noise spikes if we search the image blindly. The mosaic area is approximately 35,000 synthesized beams. With this number of independent pixels, one expects $>1$ noise spike above the $4 \sigma$ level (assuming Gaussian noise). We therefore use a $5 \sigma$ detection limit, at which level $\ll 1$ pixels are expected to show noise spikes. Because the noise varies greatly across the map, we calculate $\sigma$ locally in small subregions of the image.

Specifically, a "local" $\sigma$ is computed in $10^{\prime \prime} \times 10^{\prime \prime}(=100 \times$ 100 pixels) boxes around each pixel in the mosaic. For our detection thresholds to be meaningful, the noise must be well characterized. However, poorly sampled extended emission leads to excess noise in the $\mathrm{BN} / \mathrm{KL}$ and $\mathrm{OMC} 1-\mathrm{S}$ regions. Moreover, the noise increases toward the edges of the unit-gain region because there are fewer overlapping mosaic pointings there. Detections in these areas should be treated with some caution.

As a test of our detection threshold, we searched the maps for false detections below the $-5 \sigma$ level. None were detected, confirming that $5 \sigma$ is a reasonable detection limit. In contrast, 28 sources were seen below the $-4 \sigma$ level (most of them toward the edges of the mosaic or in the $\mathrm{BN} / \mathrm{KL}$ and $\mathrm{OMC} 1-\mathrm{S}$ regions), demonstrating that $4 \sigma$ is not a sufficiently stringent detection

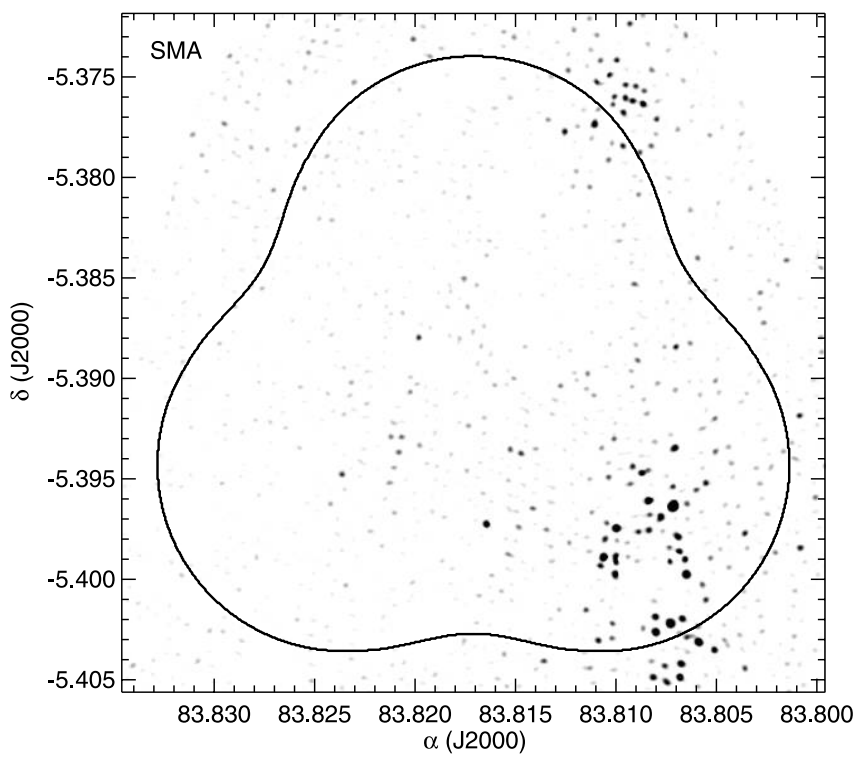

FIG. 3.-ONC imaged in $\lambda 1.3 \mathrm{~mm}$ continuum with the SMA. Only data observed on long baselines $\left(r_{u-v}>70 \mathrm{k} \lambda\right)$ were used to create the image. The unitgain region of the mosaic is indicated by a solid curve. The synthesized beam has a roughly circularly symmetric core with a FWHM of $0.98^{\prime \prime}$. The rms within the unitgain contour varies from 0.8 to $28 \mathrm{mJy}$, with a mean value of $2.7 \mathrm{mJy}$. The BN/KL and $\mathrm{OMC} 1-\mathrm{S}$ regions produce the strong emission visible in the upper and lower right corners of the map. The map has been divided by the theoretical rms to visually downweight the noisier edges.

threshold (and that the noise across our mosaic is not always Gaussian)

Instead of blindly searching for detections, we can also use our prior knowledge of the locations of near-IR cluster members and search only these positions. For these $\sim 250$ predetermined positions, $\sim 0.3$ sources are expected to show emission above the $3 \sigma$ level from Gaussian noise. We can therefore try a $3 \sigma$ detection threshold, where $\sigma$ is the noise determined locally (as above) in $10^{\prime \prime} \times 10^{\prime \prime}$ subregions centered on individual cluster member positions. Although the noise in the mosaic is not always Gaussian, this $3 \sigma$ threshold appears reasonable; none of the nearIR source positions were detected below the $-3 \sigma$ level.

Sources with $1 \mathrm{~mm}$ continuum emission at the $>3 \sigma$ level in our maps are deemed to coincide with near-IR cluster members if the millimeter peaks and near-IR source positions lie within $0.4^{\prime \prime}$ of each other. The estimated relative positional accuracy of $0.4^{\prime \prime}$ is the quadrature sum of uncertainties from centroiding the millimeter images $\left(\sim 0.5 \theta_{\text {beam }} /\right.$ signal-to-noise $\left.\approx 0.1^{\prime \prime}\right)$, uncertainties in the absolute astrometry due to baseline errors $\left(\sim 0.2^{\prime \prime}\right)$, and uncertainties in the near-IR source positions $\left(\sim 0.3^{\prime \prime}\right)$.

We detected 19 sources within the unit-gain contour of our mosaic above the $5 \sigma$ level (Table 1). Of these, 12 are coincident with near-IR cluster members listed in Hillenbrand \& Carpenter (2000). An additional 21 objects were detected above the $3 \sigma$ level toward positions of near-IR sources. Images of sources detected in our mosaic in $1 \mathrm{~mm}$ continuum are displayed in Figure 5.

While the BN object was detected, we defer discussion of this high-mass, embedded object (e.g., Gezari et al. 1998; Plambeck et al. 1995) to a later paper that will examine the $\mathrm{BN} / \mathrm{KL}$ region in detail. ${ }^{4}$ In the remaining discussion, we focus our attention

\footnotetext{
${ }^{4}$ Although Source I is detected as a strong, individual object in our CARMA $B$ array data, we do not detect it in our combined SMA+CARMA mosaic because of confusion with the hot core. Source I will also be discussed in the later paper.
} 


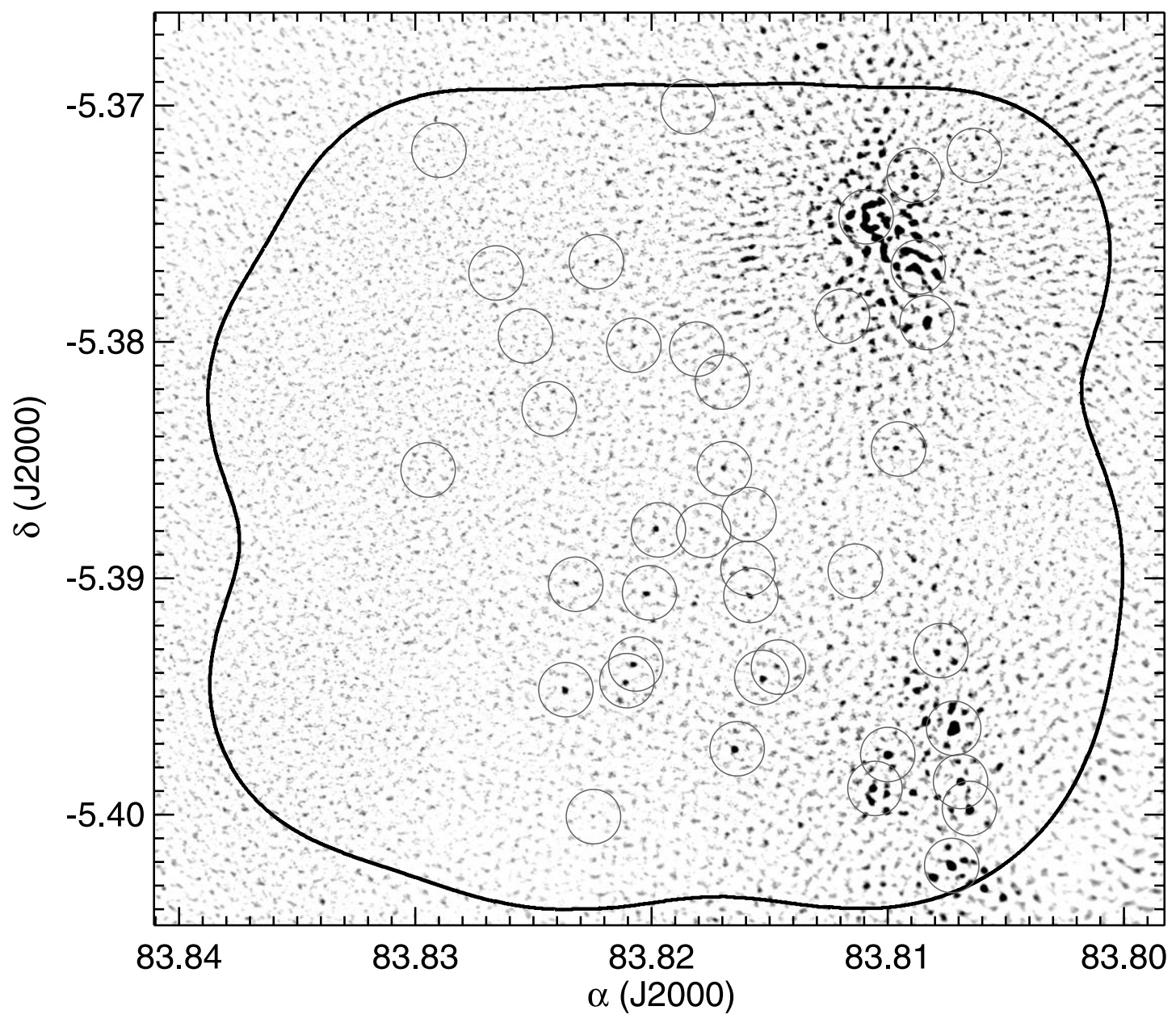

FIG. 4.-ONC imaged in $\lambda 1.3 \mathrm{~mm}$ continuum with CARMA and the SMA (grayscale). CARMA and SMA data were combined in the $u-v$ plane, and only data observed on long baselines $\left(r_{u^{-}}>70 \mathrm{k} \lambda\right)$ were used to create this image. The angular resolution is $\sim 0.7^{\prime \prime} \times 0.6^{\prime \prime}$. The unit-gain region of the mosaic encompasses a $2^{\prime} \times 2^{\prime}$ area, as indicated by the solid contour, and the rms residuals $(1 \sigma)$ within this region vary from $1.8 \mathrm{mJy}$ in regions devoid of bright emission to $\gtrsim 10 \mathrm{mJy}$ in the crowded regions toward the right of the map. Millimeter detections above the $3 \sigma$ level coincident with near-IR cluster members and proplyds, and sources without counterparts detected above the $5 \sigma$ level $(\S 3.1)$, are indicated by open circles. The map has been divided by the theoretical rms to visually downweight the noisier edges.

on the sources detected at both infrared and millimeter wavelengths. While most of these objects do not have known stellar masses, they are likely to be low-mass stars, a conclusion based on the stellar-mass distribution computed statistically for the ONC as a whole (Hillenbrand \& Carpenter 2000).

\subsection{Angular Sizes of Detected Objects}

For each source detected in our $1 \mathrm{~mm}$ mosaic, we fitted a twodimensional elliptical Gaussian to the emission. The synthesized, clean, beam for the combined mosaic is a two-dimensional Gaussian with a FWHM of $0.69^{\prime \prime} \times 0.60^{\prime \prime}$ at a position angle (north of west) of $72^{\circ}$. At the assumed $400 \mathrm{pc}$ distance to the ONC, the core of the synthesized beam has dimensions of $240 \times 280 \mathrm{AU}$ (again at a P.A. of $72^{\circ}$ ). For simplicity, we approximate this as $240 \mathrm{AU}$ in the east-west direction and $280 \mathrm{AU}$ in the north-south direction.

For sources detected at a signal-to-noise ratio of $\sim 5$, the statistical uncertainty in the fitted FWHM is $\sim 10 \%$. Baseline errors or phase noise in our mosaics can broaden the apparent source sizes, however. We assume that a source is resolved only if the major or minor axis of the fitted FWHM is $25 \%$ larger than that of the synthesized beam. Objects for which the fitted Gaussian FWHM is smaller are considered to be unresolved.

Approximately $25 \%(9 / 39)$ of detected sources are spatially resolved in our images (Table 2). An additional nine objects (all proplyds) have been spatially resolved with the Hubble Space Telescope (HST) (Vicente \& Alves 2005). Thus, angular sizes are available for $\sim 50 \%$ of our sources. The inferred radii for resolved sources range from $\sim 90$ to $\sim 220 \mathrm{AU}$. For unresolved objects, we can say only that the emission is confined to radii smaller than $\sim 100$ AU. ${ }^{5}$ For a sample of 134 proplyds with sizes measured with $H S T$, the mean disk radius is $71 \mathrm{AU}$ (Vicente \& Alves 2005). Since the mean disk diameter is $\sim 1 / 2$ the size of the linear resolution of our observations, it is not surprising that most of the sources detected in the $1 \mathrm{~mm}$ mosaic are unresolved.

\subsection{Distinguishing Dust and Free-Free Emission}

Since we are interested in using our observations to constrain the mass of circumstellar dust around our sources, we must account for potential contributions to the observed fluxes from sources other than dust emission. Free-free emission arises in hot ionized gas, and in the ONC such conditions may exist either in $\mathrm{H}$ II regions around high-mass stars (e.g., Garay et al. 1987; Plambeck et al. 1995) or in the outer regions of disks or envelopes that are irradiated by the hot Trapezium stars (e.g., O’Dell et al. 1993;

\footnotetext{
${ }^{5}$ Larger sources, when convolved with the synthesized beam, would produce measured sizes $>25 \%$ broader than the beam.
} 
TABLE 1

Long-Wavelength Fluxes for Sources Detected in $\lambda 1.3 \mathrm{~mm}$ Continuum

\begin{tabular}{|c|c|c|c|c|c|c|c|c|c|c|}
\hline ID & $\begin{array}{c}\text { R.A. } \\
\text { (J2000.0) }\end{array}$ & $\begin{array}{c}\text { decl. } \\
\text { (J2000.0) }\end{array}$ & $\begin{array}{c}S_{880 \mu \mathrm{m}} \\
(\mathrm{mJy})\end{array}$ & $\begin{array}{c}S_{1.3 \mathrm{~mm}} \\
(\mathrm{mJy})\end{array}$ & $\begin{array}{l}S_{3 \mathrm{~mm}} \\
(\mathrm{mJy})\end{array}$ & $\begin{array}{c}S_{3.6 \mathrm{~mm}} \\
(\mathrm{mJy})\end{array}$ & $\begin{array}{l}S_{1.3 \mathrm{~cm}} \\
(\mathrm{mJy})\end{array}$ & $\begin{array}{l}S_{2 \mathrm{~cm}} \\
(\mathrm{mJy})\end{array}$ & $\begin{array}{l}S_{6 \mathrm{~cm}} \\
(\mathrm{mJy})\end{array}$ & $\begin{array}{l}S_{20 \mathrm{~cm}} \\
(\mathrm{mJy})\end{array}$ \\
\hline $147-323 \ldots \ldots \ldots \ldots \ldots \ldots$ & 53514.73 & -52322.91 & & $7.5 \pm 2.2$ & $<5.3$ & & & & & \\
\hline $158-323 \ldots \ldots \ldots \ldots \ldots \ldots$ & 53515.82 & -52322.50 & & $9.5 \pm 2.3$ & $<9.7$ & $11.4 \pm 2.0$ & $10.6 \pm 2.9$ & $11.2 \pm 1.5$ & $10.6 \pm 4.8$ & $7.3 \pm 0.7$ \\
\hline 158-327 .................... & 53515.79 & -52326.61 & & $12.8 \pm 2.4$ & $18.6 \pm 3.2$ & & & $9.2 \pm 1.4$ & $13.0 \pm 5.7$ & $7.5 \pm 1.3$ \\
\hline $159-350 \ldots \ldots \ldots \ldots \ldots \ldots$ & 53515.93 & -52349.96 & & $42.7 \pm 3.7$ & $11.9 \pm 3.3$ & $13.1 \pm 2.0$ & & $10.5 \pm 4.2$ & $16.2 \pm 5.7$ & $7.6 \pm 0.8$ \\
\hline 168-326NS ................ & 53516.82 & $\begin{array}{lll}-5 & 2326.21\end{array}$ & & $13.6 \pm 2.3$ & $<7.6$ & $10.1 \pm 2.0$ & $14.8 \pm 5.0$ & $8.4 \pm 0.3$ & & \\
\hline 170-337 .................. & 53516.96 & -52337.04 & $38.1 \pm 5.2$ & $12.9 \pm 2.2$ & $<5.9$ & & & $6.6 \pm 2.4$ & $13.6 \pm 3.3$ & $6.8 \pm 0.7$ \\
\hline $171-340$.................... & 53517.05 & -52339.59 & $18.3 \pm 4.6$ & $13.0 \pm 2.3$ & $<6.1$ & & & & & \\
\hline 177-341 ..................... & 53517.67 & -52340.96 & & $15.8 \pm 2.1$ & $16.7 \pm 2.8$ & & & $10.8 \pm 3.7$ & $14.4 \pm 4.0$ & $7.7 \pm 0.7$ \\
\hline 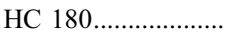 & 53517.39 & -5240.30 & & $10.7 \pm 3.0$ & $<4.6$ & & & & & \\
\hline HC $189 \ldots \ldots \ldots \ldots \ldots . .$. & 53514.53 & -52356.00 & & $99.6 \pm 8.4$ & $<9.1$ & & & & & \\
\hline 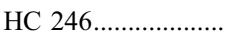 & 53515.68 & -52339.10 & & $17.8 \pm 2.4$ & $<7.5$ & & & & & \\
\hline 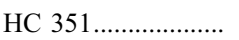 & 53519.07 & -5237.50 & & $8.7 \pm 2.8$ & $<4.6$ & & & & & \\
\hline 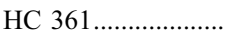 & 53514.29 & -5234.30 & & $19.2 \pm 3.2$ & $<7.6$ & & & & & \\
\hline HC $383 \ldots \ldots \ldots \ldots \ldots$ & 53517.84 & -52258.20 & & $7.0 \pm 2.3$ & $<5.2$ & & & & & \\
\hline HC 401.................... & 53516.08 & -52254.10 & & $7.3 \pm 2.1$ & $<5.6$ & & & & & \\
\hline 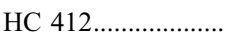 & 53516.34 & -52249.10 & & $7.7 \pm 2.3$ & $<5.6$ & & & & & \\
\hline 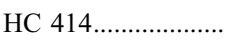 & 53516.98 & -52248.50 & & $9.1 \pm 2.2$ & $<5.2$ & & & $3.8 \pm 0.9$ & $7.0 \pm 5.6$ & $10.5 \pm 0.5$ \\
\hline 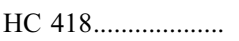 & 53518.08 & -52247.10 & & $7.6 \pm 2.5$ & $<5.5$ & & & & & \\
\hline 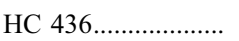 & 53518.38 & -52237.50 & & $8.7 \pm 2.8$ & $<5.5$ & & $16.6 \pm 1.3$ & $9.0 \pm 0.3$ & & $11.2 \pm 0.4$ \\
\hline HC $438 \ldots \ldots \ldots \ldots \ldots \ldots . .$. & 53514.09 & -52236.60 & & $67.8 \pm 14.2$ & $<12.1$ & & & $2.0 \pm 0.2$ & & \\
\hline HC 440...................... & 53517.36 & -52235.80 & & $13.0 \pm 2.8$ & $<7.0$ & & & & & \\
\hline 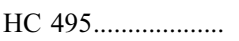 & 53513.52 & $\begin{array}{lll}-5 & 22 & 19.60\end{array}$ & & $22.1 \pm 6.6$ & $<6.4$ & & & & & \\
\hline HC 498...................... & 53518.96 & $\begin{array}{lll}-5 & 22 & 18.80\end{array}$ & & $15.3 \pm 4.6$ & $<4.3$ & & & & & \\
\hline HC 514....................... & 53516.43 & $\begin{array}{lll}-5 & 22 & 12.20\end{array}$ & & $23.7 \pm 7.4$ & $<4.6$ & & & & & \\
\hline
\end{tabular}

NoтеS.-Units of right ascension are hours, minutes, and seconds, and units of declination are degrees, arcminutes, and arcseconds. All quoted uncertainties are $1 \sigma$; uncertainties (of $10 \%$ ) in the overall flux scale are not included in this table. The MM sources are detected only at wavelengths $\gtrsim 1 \mathrm{~mm}$. HC objects are known near-IR cluster members from Hillenbrand \& Carpenter (2000) that are also detected at millimeter wavelengths, and the sources with numerical labels are a subset of this sample that are also detected optically as proplyds (e.g., O’Dell et al. 1993). LMLA 162 is absent from Hillenbrand \& Carpenter (2000) but seen at $3.6 \mu$ m by Lada et al. (2004); we also see a faint $2 \mu \mathrm{m}$ point source at this location in a 2MASS image. MM8 and MM13 were detected by Eisner \& Carpenter (2006), and MM21 was detected by Eisner \& Carpenter (2006) but associated with HC 178 (we find that MM21 and HC 178 are separated by an angle larger than our relative positional uncertainties). LMLA 162, MM8, MM13, MM21, and MM22 were also detected in previous $1.3 \mathrm{~mm}$ observations by Zapata et al. (2005). MM23 and MM24 are newly detected here.

REFERENCES.-References for the fluxes are as follows: $880 \mu \mathrm{m}$ (Williams et al. 2005); $1.3 \mathrm{~mm}$ (this work); $3 \mathrm{~mm}$ (Eisner \& Carpenter 2006); $3.6 \mathrm{~mm}$ (Mundy et al. 1995); $1.3 \mathrm{~cm}$ (Zapata et al. 2004); 2 and $6 \mathrm{~cm}$ (Felli et al. 1993b); $20 \mathrm{~cm}$ (Felli et al. 1993a). Quoted upper limits for the $3 \mathrm{~mm}$ fluxes are $3 \sigma$.

Henney \& O'Dell 1999). While some sources in the ONC have shown flares of cyclotron emission (e.g., Bower et al. 2003; Furuya et al. 2003), we expect that such rare events will not contribute significantly to the $1.3 \mathrm{~mm}$ fluxes, and we do not consider them here.

Because the spectral shape of free-free radiation differs from that of thermal dust emission, comparing $1 \mathrm{~mm}$ measurements with longer wavelength data enables us to distinguish these components. We use long-wavelength fluxes from the literature (Felli et al. 1993a, 1993b; Mundy et al. 1995; Zapata et al. 2004; Eisner \& Carpenter 2006; Forbrich et al. 2008). In addition, we use $880 \mu \mathrm{m}$ fluxes measured by Williams et al. (2005) for the few objects where these are available.
For a freely expanding, fully ionized wind with constant $\dot{M}$, such as we expect for proplyds, free-free emission will have the following spectrum: ${ }^{6}$

$$
F_{\nu, \mathrm{ff}}=\left\{\begin{array}{cc}
F_{\nu, \text { turn }}\left(\nu / \nu_{\text {turn }}\right)^{-0.1} & \nu \geq \nu_{\text {turn }} \\
F_{\nu, \text { turn }}\left(\nu / \nu_{\text {turn }}\right)^{0.6} & \nu \leq \nu_{\text {turn }}
\end{array}\right.
$$

\footnotetext{
${ }^{6}$ In a previous paper (Eisner \& Carpenter 2006), we assumed that free-free emission originated from static $\mathrm{H}$ II regions rather than from winds, as in the present work. This choice affects only the long-wavelength behavior of the freefree spectrum, and is relatively unimportant to our analysis.
} 

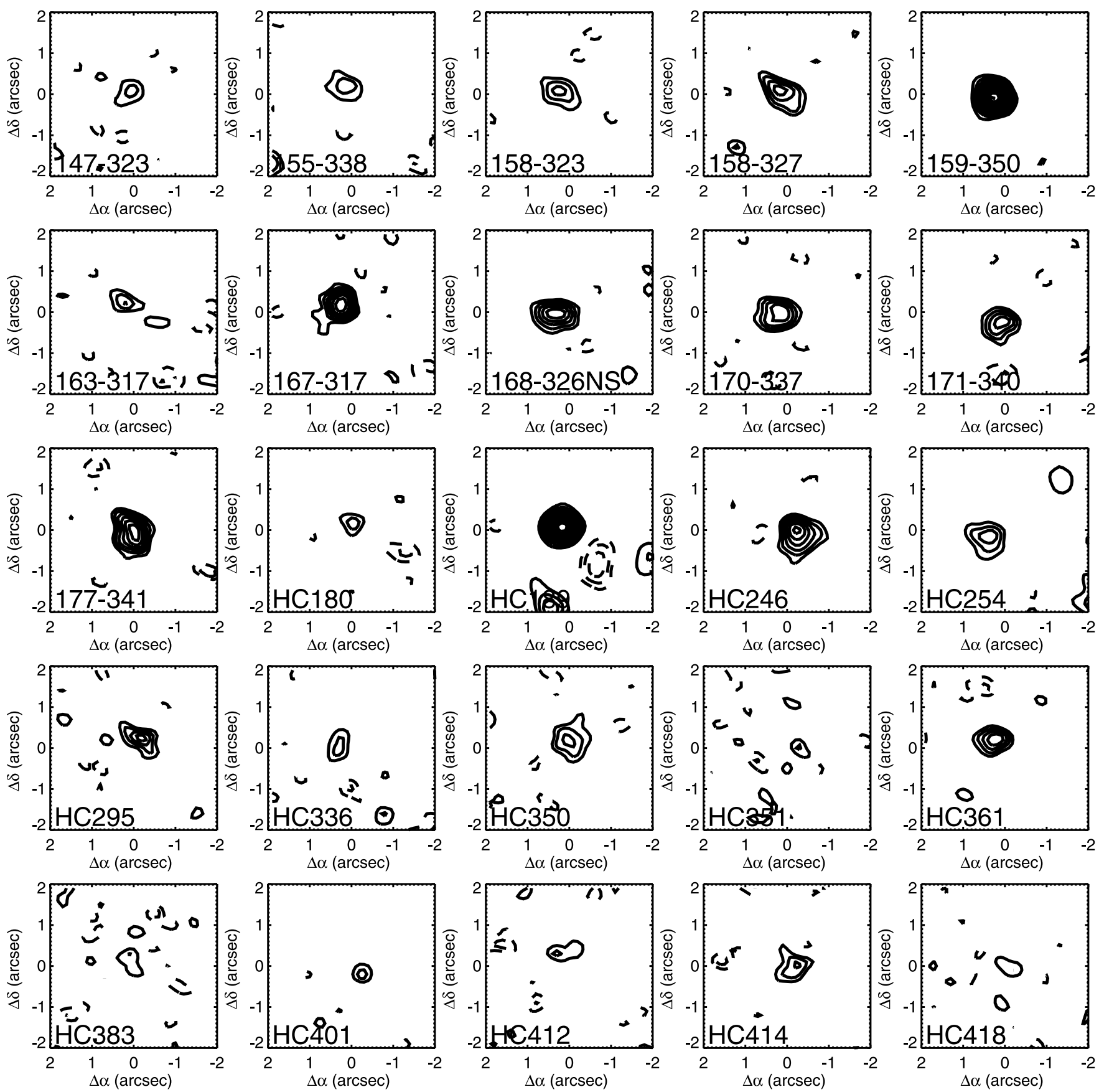

FIG. 5.-Contour images of sources detected in $1 \mathrm{~mm}$ continuum emission. Contour increments are $1 \sigma$, beginning at $\pm 2 \sigma$, where $\sigma$ is determined locally for each object

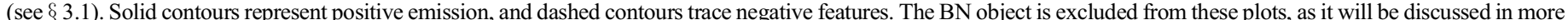

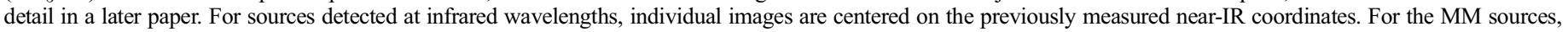
detected only at $\gtrsim 1 \mathrm{~mm}$ wavelengths, images are centered on the peak fluxes.

Here, $\nu_{\text {turn }}$ is the frequency above which the wind is optically thin at all radii. We include a derivation of this result in the Appendix, and an alternative derivation can be found in Wright \& Barlow (1975).

For $\nu<\nu_{\text {turn }}$, the inner parts of the wind are optically thick to free-free radiation. If we adopt a simple model for proplyd winds where $\dot{M}=10^{-7} M_{\odot} \mathrm{yr}^{-1}$, with a spherical wind velocity of $20 \mathrm{~km} \mathrm{~s}^{-1}$ (e.g., Henney \& O'Dell 1999) and $T_{e}=10^{4} \mathrm{~K}$, we can estimate the size of the optically thick region. Using equation (A5), we obtain $x_{\tau \approx 1} \sim 1$ AU at $\lambda 1 \mathrm{~mm}$ and $\sim 30$ AU at $\lambda 10 \mathrm{~cm}$. Even at $10 \mathrm{~cm}$, this is smaller than the likely windlaunching regions for proplyds, and free-free emission from most proplyds is likely to be fully optically thin. For the highest measured mass-loss rates of $\sim 10^{-6} M_{\odot} \mathrm{yr}^{-1}$ (e.g., Henney \& O'Dell 1999), the optically thick regions of proplyd winds are $\sim 130 \mathrm{AU}$ for $\lambda \sim 10 \mathrm{~cm}$. We therefore do not expect to see a spectral turnover (eq. [1]) for wavelengths $\lesssim 5 \mathrm{~cm}$.

Emission from cool dust is added to this free-free emission to obtain a model of the observed flux. We assume that

$$
\begin{aligned}
F_{\nu, \text { dust }} & =F_{230 \mathrm{GHz}, \text { dust }}(\nu / 230 \mathrm{GHz})^{2+\beta} \\
& =F_{230 \mathrm{GHz}, \text { dust }}(\nu / 230 \mathrm{GHz})^{3},
\end{aligned}
$$



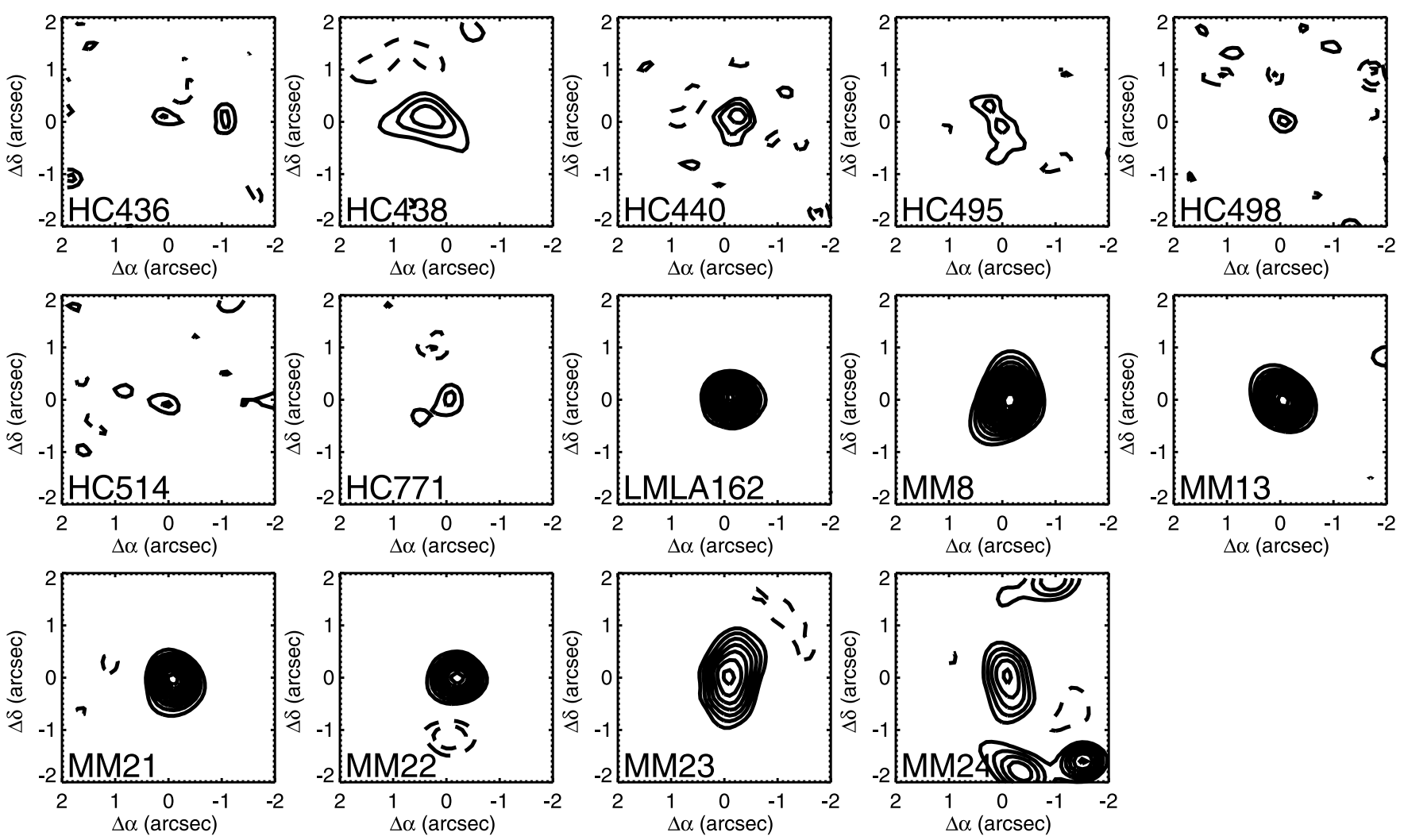

FIg. 5-Continued

where $\beta=1$ (e.g., Beckwith et al. 1990). Other values of $\beta$ cannot be ruled out based on our data for most objects, and $\beta=0$, corresponding to emission from optically thick or large-grained dust, is typically compatible with the data.

We estimate the relative contributions of dust and free-free emission by fitting this model, $F_{\nu}=F_{\nu \text {,ff }}+F_{\nu \text {, dust }}$, to our measured $1.3 \mathrm{~mm}$ fluxes and to $880 \mu \mathrm{m}, 3 \mathrm{~mm}, 3.6 \mathrm{~mm}, 1.3 \mathrm{~cm}, 2 \mathrm{~cm}$, $6 \mathrm{~cm}$, and $20 \mathrm{~cm}$ fluxes from the literature. For comparison, we also fit a dust-only model, described by equation (2), with $\beta=1$ and $\beta=0$. For objects detected at centimeter wavelengths, we fit the dust + free-free model to the $\geq 4$ flux measurements for each source, and thus we are able to determine the three free parameters of the model, $\nu_{\text {turn }}, F_{\nu \text {,turn }}$, and $F_{\nu \text {,dust }}$. For sources with $\leq 3$ flux measurements (i.e., those undetected in centimeter-wavelength surveys), we fit only the dust-emission model to the data.

Given the noise level of previous centimeter observations covering the entire region of our $1 \mathrm{~mm}$ mosaic $(\lesssim 0.3 \mathrm{mJy}$; Felli et al. 1993a, 1993b) and the measured $1 \mathrm{~mm}$ fluxes for detected objects ( $\gtrsim 10 \mathrm{mJy}$ ), sources undetected at centimeter wavelengths are probably dominated by dust emission. For a source with $10 \mathrm{mJy}$ flux at $1 \mathrm{~mm}$, a nondetection at $10 \mathrm{~cm}$ implies that $\lesssim 0.2 \mathrm{mJy}$, or $\lesssim 2 \%$, of the measured $1 \mathrm{~mm}$ flux is due to free-free emission. For simplicity, we attribute $100 \%$ of the $1 \mathrm{~mm}$ fluxes to dust emission for these objects.

Fluxes, from submillimeter to radio wavelengths, and models are plotted in Figure 6, and the fluxes due to thermal dust emission are listed in Table 2. Uncertainties for these dust fluxes are given by the $1 \sigma$ uncertainties of the model fits. The majority of detected sources appear to be dominated by dust emission. However, for the subset of the sample seen in optical emission or absorption with HST (the proplyds at the top of Table 1), the $1 \mathrm{~mm}$ fluxes are dominated by free-free emission. This probably reflects the relative proximity of proplyds to the luminous Trapezium stars.

\subsection{Estimating Circumstellar Dust Masses}

The mass of circumstellar dust is related to the component of the $1 \mathrm{~mm}$ continuum flux due to dust emission. Assuming the dust is optically thin and following Hildebrand (1983),

$$
M_{\text {dust }}=\frac{S_{\nu, \text { dust }} d^{2}}{\kappa_{\nu, \text { dust }} B_{\nu}\left(T_{\text {dust }}\right)} \text {. }
$$

Here, $\nu$ is the observed frequency, $S_{\nu \text {, dust }}$ is the observed flux due to cool dust, $d$ is the distance to the source, $\kappa_{\nu \text {, dust }}=\kappa_{0}\left(\nu / \nu_{0}\right)^{\beta}$ is the dust-mass opacity, $T_{\text {dust }}$ is the dust temperature, and $B_{\nu}$ is the Planck function. We assume that $d \approx 400 \mathrm{pc}, \kappa_{0}=0.0002 \mathrm{~cm}^{2} \mathrm{~g}^{-1}$ at $1.3 \mathrm{~mm}, \beta=1.0$ (Hildebrand 1983; Beckwith et al. 1990), and $T_{\text {dust }}=20 \mathrm{~K}$ (based on the average dust temperature inferred for Taurus; Andrews \& Williams 2005; see also the discussion in Carpenter 2002; Williams et al. 2005). The dust mass can be converted into a total circumstellar mass by assuming the canonical gas-to-dust mass ratio, $M_{\text {circumstellar }}=M_{\text {dust }} \times 100$. Column (3) of Table 2 lists the estimated circumstellar masses for detected objects.

Uncertainties in the assumed values of these parameters (notably $\kappa$ ) lead to uncertainties in the derived masses (in an absolute sense) of at least a factor of 3 (e.g., Pollack et al. 1994), which are not included in the uncertainties listed in the table. Values of $\kappa_{\nu \text {, dust }}$ and $T_{\text {dust }}$ may also vary across our sample. Since the cluster population in Orion is roughly coeval (e.g., Hillenbrand 1997), such effects should be minimal. However, there is some spread in stellar masses, which may lead to some range in these parameters. For example, since some of the objects in Table 1 may be massive stars, the millimeter flux may contain contributions from dust hotter than the assumed $20 \mathrm{~K}$; if $T_{\text {dust }}=30 \mathrm{~K}$, then the computed dust masses would be lower by a factor of 1.6. For the predominantly low-mass cluster population (Hillenbrand 
TABLE 2

Derived Quantities for Detected Sources

\begin{tabular}{|c|c|c|c|c|}
\hline $\begin{array}{l}\text { ID } \\
\text { (1) }\end{array}$ & $\begin{array}{c}S_{\nu, \text { dust }} \\
\text { (mJy) } \\
(2)\end{array}$ & $\begin{array}{c}M_{\text {circumstellar }} \\
\left(M_{\odot}\right) \\
(3)\end{array}$ & $\begin{array}{c}R_{\text {disk }} \\
\text { (AU) } \\
\text { (4) }\end{array}$ & $\begin{array}{l}S_{\nu, \tau} \gtrsim 1 \\
(\mathrm{mJy}) \\
(5)\end{array}$ \\
\hline $147-323 \ldots \ldots \ldots \ldots \ldots$ & $7.5 \pm 2.2$ & $0.009 \pm 0.003$ & 88 & 83 \\
\hline $155-338$ … & $4.0 \pm 4.0$ & $0.005 \pm 0.005$ & 102 & 112 \\
\hline $158-323 \ldots \ldots \ldots \ldots \ldots \ldots$ & $1.0 \pm 1.0$ & $0.001 \pm 0.001$ & 105 & 119 \\
\hline $158-327$.................. & $5.0 \pm 4.0$ & $0.006 \pm 0.005$ & 122 & 161 \\
\hline $159-350$................. & $33.0 \pm 7.0$ & $0.042 \pm 0.009$ & 152 & 250 \\
\hline 163-317 ….............. & $6.0 \pm 2.0$ & $0.008 \pm 0.003$ & 93 & 93 \\
\hline $167-317$...................... & $2.0 \pm 2.0$ & $0.003 \pm 0.003$ & 122 & 161 \\
\hline 168-326NS ............. & $7.0 \pm 4.0$ & $0.009 \pm 0.005$ & $<100$ & $<108$ \\
\hline 170-337 ….............. & $10.0 \pm 2.5$ & $0.013 \pm 0.003$ & 126 & 171 \\
\hline $171-340 \ldots \ldots \ldots \ldots \ldots . .$. & $13.0 \pm 2.3$ & $0.016 \pm 0.003$ & 80 & 69 \\
\hline 177-341 ................... & $4.0 \pm 4.0$ & $0.005 \pm 0.005$ & 177 & 339 \\
\hline 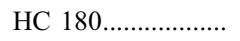 & $10.7 \pm 3.0$ & $0.013 \pm 0.004$ & $<100$ & $<108$ \\
\hline HC $189 \ldots \ldots \ldots \ldots \ldots \ldots$ & $99.5 \pm 8.4$ & $0.125 \pm 0.011$ & $<100$ & $<108$ \\
\hline HC $246 \ldots \ldots \ldots \ldots \ldots$ & $17.8 \pm 2.4$ & $0.022 \pm 0.003$ & $<100$ & $<108$ \\
\hline 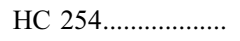 & $17.7 \pm 3.8$ & $0.022 \pm 0.005$ & $<100$ & $<108$ \\
\hline 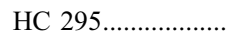 & $8.0 \pm 3.5$ & $010 \pm 0.004$ & 187 & 381 \\
\hline 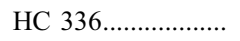 & $0.0 \pm 0.0$ & $0.000 \pm 0.000$ & $<100$ & $<108$ \\
\hline 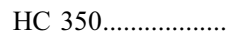 & $5.0 \pm 3.5$ & $0.006 \pm 0.004$ & $<100$ & $<108$ \\
\hline 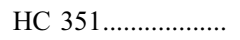 & $8.7 \pm 2.8$ & $0.011 \pm 0.003$ & $<100$ & $<108$ \\
\hline 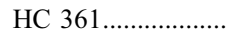 & $19.2 \pm 3.2$ & $0.024 \pm 0.004$ & $<100$ & $<108$ \\
\hline 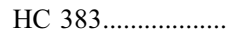 & $7.0 \pm 2.3$ & $0.009 \pm 0.003$ & 202 & 441 \\
\hline 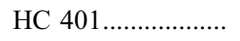 & $7.3 \pm 2.1$ & $0.009 \pm 0.003$ & $<100$ & $<108$ \\
\hline 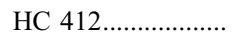 & $7.7 \pm 2.3$ & $0.010 \pm 0.003$ & 161 & 283 \\
\hline 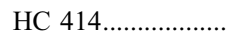 & $3.0 \pm 3.0$ & $0.004 \pm 0.004$ & $<100$ & $<108$ \\
\hline 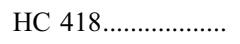 & $7.6 \pm 2.5$ & $0.010 \pm 0.003$ & $<100$ & $<108$ \\
\hline 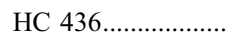 & $1.0 \pm 1.0$ & $0.001 \pm 0.001$ & $<100$ & $<108$ \\
\hline 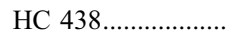 & $67.8 \pm 14.2$ & $0.085 \pm 0.018$ & 200 & 433 \\
\hline 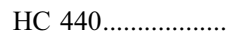 & $13.0 \pm 2.8$ & $0.016 \pm 0.004$ & $<100$ & $<108$ \\
\hline 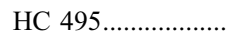 & $22.1 \pm 6.6$ & $0.028 \pm 0.008$ & $<100$ & $<108$ \\
\hline 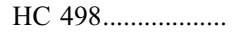 & $15.3 \pm 4.6$ & $0.019 \pm 0.006$ & $<100$ & $<108$ \\
\hline 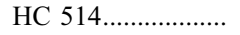 & $23.7 \pm 7.4$ & $0.030 \pm 0.009$ & 166 & 299 \\
\hline 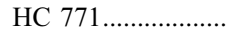 & $16.1 \pm 4.9$ & $0.020 \pm 0.006$ & 176 & 337 \\
\hline LMLA $162 \ldots \ldots \ldots \ldots$ & $103.3 \pm 7.2$ & $0.130 \pm 0.009$ & $<100$ & $<108$ \\
\hline 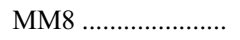 & $407.2 \pm 27.5$ & $0.512 \pm 0.035$ & $<100$ & $<108$ \\
\hline MM13 ...................... & $317.5 \pm 25.1$ & $0.399 \pm 0.032$ & $<100$ & $<108$ \\
\hline MM21 ..................... & $153.6 \pm 13.2$ & $0.193 \pm 0.017$ & $<100$ & $<108$ \\
\hline MM22 .................... & $123.0 \pm 12.7$ & $0.155 \pm 0.016$ & $<100$ & $<108$ \\
\hline MM23 ..................... & $61.2 \pm 7.4$ & $0.077 \pm 0.009$ & $<100$ & $<108$ \\
\hline MM24 .................... & $167.3 \pm 27.0$ & $0.211 \pm 0.034$ & 217 & 514 \\
\hline
\end{tabular}

Notes. $-S_{\nu \text {, dust }}$ is the component of the observed $1.3 \mathrm{~mm}$ emission due to cool dust, determined from a fit to long-wavelength fluxes of a model including thermal free-free emission as well as dust emission (see Fig. 6). Quoted uncertainties are $1 \sigma$ and do not include systematic uncertainties associated with the overall flux calibration or conversion from flux to mass.

$\&$ Carpenter 2000), from which the sources listed in Table 1 are drawn, the assumed values for $\kappa_{\nu \text {, dust }}$ and $T_{\text {dust }}$ presumably do not vary much, and the masses predicted by equation (3) are probably valid in a relative sense to within a factor of 2 .

\subsection{Constraints on Dust Optical Depth}

We perform a simple test to determine whether the optical depth $(\tau)$ might become comparable to or larger than unity in the systems under study. Using disk sizes measured either from our data or HST data (Vicente \& Alves 2005), or limits on disk sizes from our observations, we compute the emission expected from optically thick dust with a temperature of $20 \mathrm{~K}$ :

$$
F_{\nu, \tau \gtrsim 1}=B_{\nu}\left(T_{\text {dust }}\right) \pi\left(\frac{R_{\text {disk }}}{d}\right)^{2} \cos i \approx 112 \mathrm{mJy}\left(\frac{R_{\text {disk }}}{100 \mathrm{AU}}\right)^{2} .
$$

Here, $R_{\text {disk }}$ is the disk radius, $i$ is the inclination, and $d$ is the distance. For simplicity, we take $i=0$, which leads to an upper limit on the flux for an optically thick disk of radius $R_{\text {disk }}$. Fluxes expected for optically thick dust for our sample are listed in Table 2.

For most objects, the fluxes (or upper limits) expected for optically thick dust are substantially higher than our measured fluxes (Table 2). These disks must be either highly inclined or optically thin. Since it is unlikely that all of the disks detected in our observations are edge-on (especially since these would be the dimmest portion of a sample of randomly inclined, optically thick disks), we take this as evidence for optically thin material. Although many of the disk sizes, and hence the expected optically thick fluxes, are upper limits, the mean radius for proplyds in Orion of $\sim 70 \mathrm{AU}$ (e.g., Vicente \& Alves 2005) would produce an optically thick flux of $\sim 50 \mathrm{mJy}$, which is still higher than the majority of our measured fluxes.

We therefore believe that most disks detected in our observation are composed largely of optically thin dust, and that the circumstellar dust masses computed in $\S 3.4$ are reasonable for these sources. There are a few among the sources detected only at $\gtrsim 1 \mathrm{~mm}$ wavelengths (MM8, MM13, and MM21) for which the measured fluxes are comparable to or larger than the expected optically thick fluxes. The dust in these objects is either optically thick or hotter than $20 \mathrm{~K}$, as may occur around higher mass (proto)stars.

\subsection{Stacking Analysis}

With the large number of young stars contained within our mosaic, we can enhance the effective sensitivity by considering the ensemble of $\sim 225$ sources not detected individually. For each known cluster member within the mosaic that is not detected above the $3 \sigma$ noise level, we make a $10^{\prime \prime} \times 10^{\prime \prime}$ subimage centered on the stellar position. We weight the subimage by the local rms (determined as described in $\S 3.1$ ), sum all of the weighted images, and divide by the sum of the weights to normalize. We exclude any cluster members known to have radio-wavelength emission (Felli et al. 1993a, 1993b).

The weighted image is shown in Figure 7. The average flux for the ensemble of nondetected sources is $0.9 \pm 0.2 \mathrm{mJy}$, with a significance of $>4 \sigma$. The peak flux is centered on the mean position of the near-IR sources (within the positional uncertainties of $\sim 0.4^{\prime \prime}$ ), and resembles the synthesized beam core, indicating that the average source is compact.

Since the positional uncertainties are comparable to the halfwidth at half-maximum of the synthesized beam, the average flux seen in Figure 7 may be slightly reduced because different sources in the ensemble do not lie exactly atop one another. Assuming the positional uncertainties are random and Gaussian distributed, one would expect a reduction in the measured average flux of $\sim 35 \%$. Correcting for the potential flux smearing, one would obtain an average flux for the ensemble of $1.2 \pm 0.2 \mathrm{mJy}$. We verify this by integrating the central region of the average image over a region with 4 times the area of the synthesized beam; as expected, we find an integrated flux of $\sim 1.2 \mathrm{mJy}$.

Low-level free-free emission may contaminate the average image and hence bias the average flux inferred for the ensemble. The $1 \sigma$ sensitivity in centimeter-wavelength surveys is $\sim 0.3 \mathrm{mJy}$ (e.g., Felli et al. 1993b). We argued in $\S 3.3$ that gaseous winds in the $\mathrm{ONC}$ are likely to be optically thin to free-free emission, and hence that $F_{\nu \text {,ff }} \propto \nu^{-0.1}$. Thus, we would expect free-free emission to be no stronger than $\sim 0.2 \mathrm{mJy}$ at $1 \mathrm{~mm}$ wavelengths for sources undetected in centimeter-wavelength surveys. This is comparable to the $1 \sigma$ sensitivity in our $1.3 \mathrm{~mm}$ average image. 

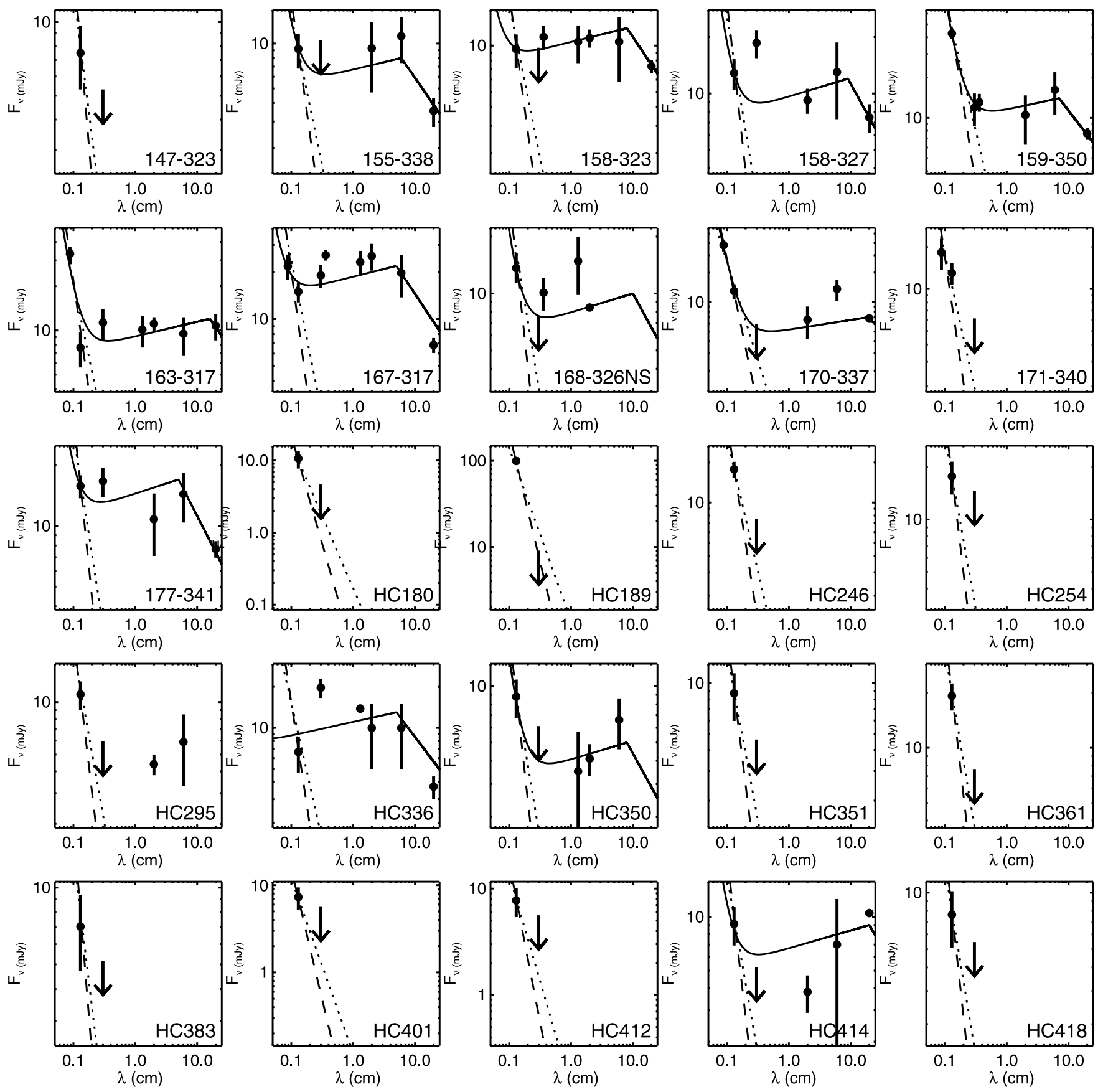

FIG. 6.-Millimeter and radio fluxes for our sample ( filled circles), along with best-fit models including free-free and thermal dust emission. Models including free-free and dust emission are indicated by solid lines, while dotted and dashed lines show dust-only models with $\beta=0$ and $\beta=1$, respectively. The free-free flux density is proportional to $\nu^{-0.1}$ for gas that is optically thin for all radii, and to $\nu^{0.6}$ for a partially optically thick gaseous wind; the emission is thus parameterized by the flux at a single wavelength and turnover frequency. The emission from cool dust is proportional to $\nu^{2+\beta}$.

Since the average image is detected above the $4 \sigma$ level, $>75 \%$ of the average flux comes from dust.

\section{DISCUSSION}

\subsection{Nature of Detected Sources}

The subarcsecond resolution of the CARMA observations is enough to marginally resolve some of the detected sources in the ONC, and in principle we could observe flattened, disklike geometries. For example, Figure 8 shows that 177-341 has a disklike morphology aligned with the silhouette disk seen by HST. While only a few sources can be well resolved with our observations,
HST observations show that many of the observed proplyds appear disklike (McCaughrean \& O’Dell 1996; Bally et al. 1998a), with some even exhibiting silhouette disks (Bally et al. 2000). For the proplyds and well-resolved millimeter sources, the $1 \mathrm{~mm}$ emission evidently arises from disklike distributions.

Mid-IR emission is also observed toward many of the sources detected at $1 \mathrm{~mm}$. 82\% of sources (all except the "MM" sources and HC 495) are also seen at $3.6 \mu \mathrm{m}$ (Lada et al. 2004), and 48\% are seen at $11.7 \mu \mathrm{m}$ (Smith et al. 2005). While $3.6 \mu \mathrm{m}$ emission may trace stellar photospheres and/or infrared excess, the $11.7 \mu \mathrm{m}$ emission provides direct evidence for circumstellar material at least out to radii of a few AU. Thus, many detected sources (the 

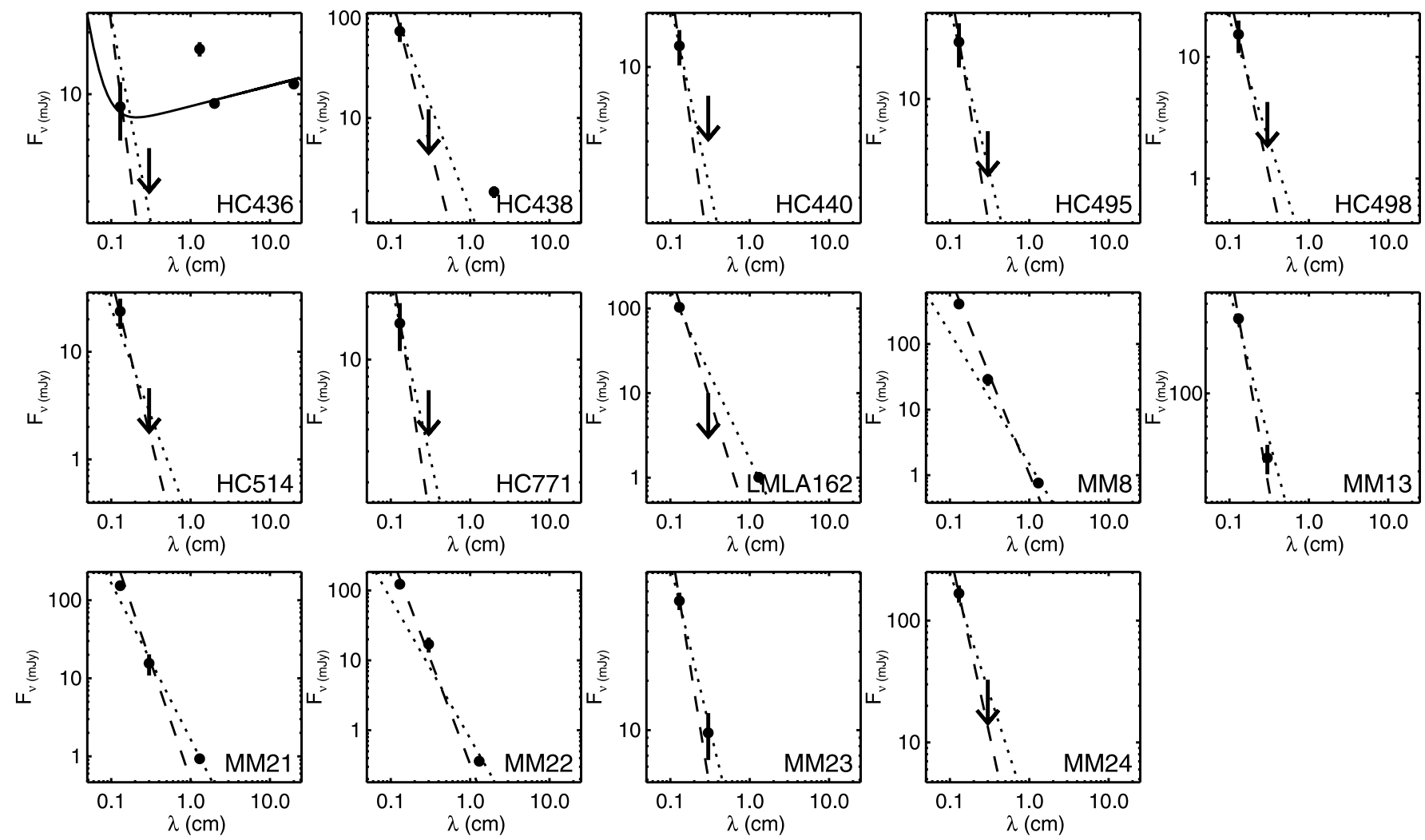

FIG. 6-Continued

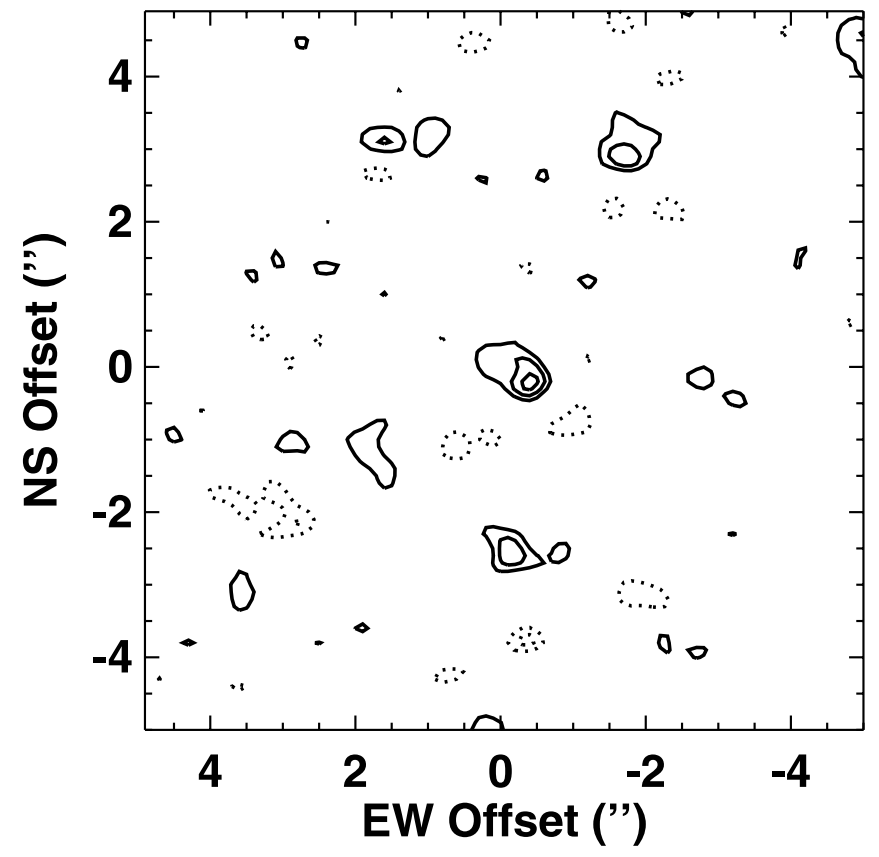

FIG. 7.-Average image, obtained by stacking the $1 \mathrm{~mm}$ continuum emission observed toward each of 226 low-mass near-IR sources not detected individually above the $3 \sigma$ level. Contour levels begin at $\pm 2 \sigma$, and the contour interval is $1 \sigma$ (negative contours are shown as dotted lines). Emission is detected for the ensemble at a significance of $\gtrsim 4 \sigma$ and exhibits a compact (and beamlike) morphology approximately centered on the origin. The degree to which the emission is smeared out is consistent with the $\sim 0.4^{\prime \prime}$ positional errors in the near-IR source positions.

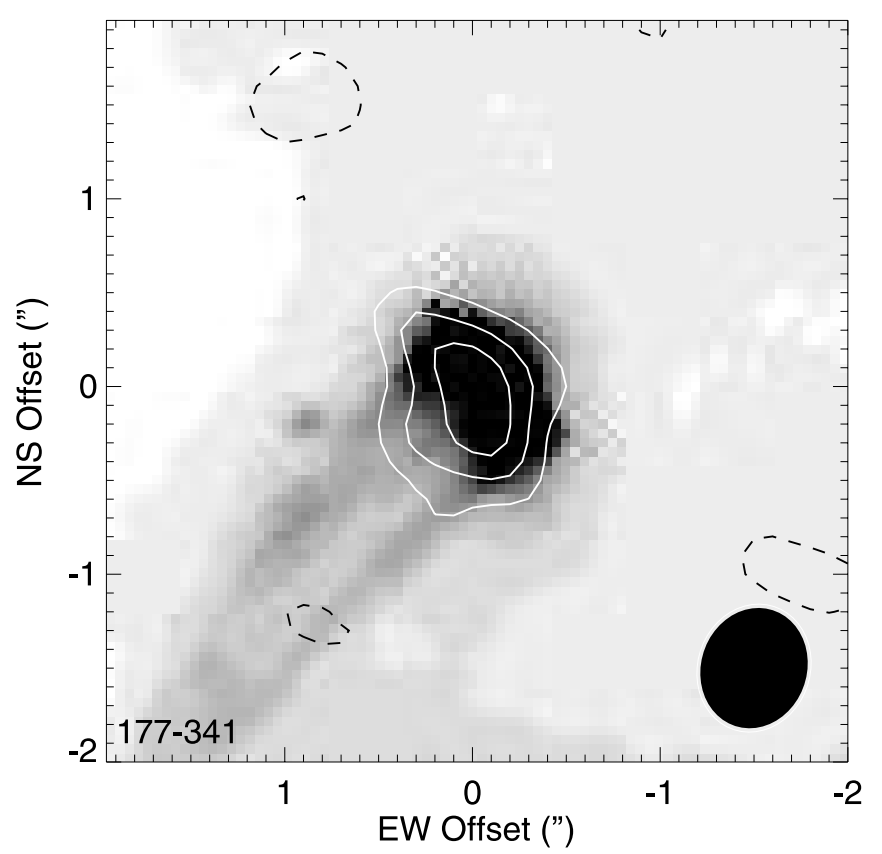

Fig. 8.-Contour image of the $1.3 \mathrm{~mm}$ wavelength continuum emission toward the proplyd 177-341 overlaid on an HST map (Bally et al. 2000) of the same position. Positive contours are solid white curves, and negative contours are dashed black curves. Contours are $\pm 2,4$, and $6 \sigma$. The FWHM of the synthesized beam is shown as a filled black oval. 
majority, if the "MM" objects are excluded ) have evidence of inner circumstellar disks.

More generally, where $1 \mathrm{~mm}$ emission is detected toward known near-IR cluster members, the fact that the near-IR light is visible despite the high extinctions $\left(A_{V} \gtrsim 300\right)$ that one would derive based on the amount of material needed to produce the $1 \mathrm{~mm}$ emission (for spherically distributed material) implies that the dust lies in flattened, disklike distributions (see also, e.g., Beckwith et al. 1990; Eisner \& Carpenter 2003).

It is interesting to speculate as to the nature of sources detected at $H K L$ bands and at millimeter wavelengths, but not at $11.7 \mu \mathrm{m}$. Of millimeter and near-IR detected sources, 13/32 (40\%) fall into this category. It is possible that some of these are transitional disks. The $H K L$ emission may trace the stellar photosphere of a late-type star while the millimeter emission traces a remnant outer disk, but large inner clearings may lead to a lack of mid-IR excess. Better coverage of the wavelength range between $10 \mu \mathrm{m}$ and $1 \mathrm{~mm}$ is needed to test this hypothesis.

For sources without near-IR detections, the "MM" sources in Table 1, the arguments presented above do not apply. Although the emission appears to trace circumstellar dust, the fact that no near-IR counterparts are observed suggests high columns of obscuring material. The MM sources all lack mid-IR counterparts as well. These objects appear to be so embedded that they are still highly obscured even at $11.7 \mu \mathrm{m}$. All of the MM sources reside in either the Orion $\mathrm{BN} / \mathrm{KL}$ or OMC1-S region, both of which are known to contain young, embedded sources, $\mathrm{H}$ II regions, and outflows (e.g., Ziurys et al. 1990; Bachiller 1996; Zapata et al. 2004).

All of the sources in OMC1-S (LMLA 162, MM8, MM13, MM21, and MM22) have been detected at $1.3 \mathrm{~mm}$ wavelength in previous observations (Zapata et al. 2005, 2007). Measured fluxes are similar to those listed in Table 1, but somewhat lower in most cases, presumably because the poorer $u-v$ coverage did not allow large negative sidelobe contributions from extended emission to be fully removed. All of these objects appear to drive molecular outflows traced by $\mathrm{CO}$ or $\mathrm{SiO}$ emission (Zapata et al. 2005, 2006).

We classify the MM sources as candidate Class 0 or Class I protostars. As discussed above, it appears that the $1.3 \mathrm{~mm}$ emission from at least some of these sources may trace dust hotter than $20 \mathrm{~K}$. Such warm dust is expected in the circumstellar environments of massive protostars, suggesting that some of the MM sources trace high-mass protostars.

Several sources detected in previous surveys were not detected here. HC 178, HC 192, HC 282, MM3, MM4, MM10, MM15, MM16, MM19, and MM20 should have been detected if their $3 \mathrm{~mm}$ fluxes (Eisner \& Carpenter 2006) traced dust emission; however, they would not have been detected if the objects exhibited flat spectra (e.g., from free-free emission). MM7, MM17, and MM18 should have been detected even if they showed flat spectra. We detect a $1.3 \mathrm{~mm}$ continuum source (MM21) near HC 178, but find it to be offset by more than the relative positional uncertainties, suggesting that the previous association of HC 178 with a $3 \mathrm{~mm}$ source was mistaken. The other $3 \mathrm{~mm}$ objects trace either nondust, potentially time-variable emission, or are spurious, caused by confusion with the BN/KL and OMC1-S regions in which they reside. Because our $1 \mathrm{~mm}$ observations have $u-v$ coverage far superior to previous observations, they are less prone to such spurious detections. One source detected by Williams et al. (2005) at $880 \mu \mathrm{m}(171-334)$ is not detected at $1.3 \mathrm{~mm}$; if the emission comes from small-grained dust, then the expected $1.3 \mathrm{~mm}$ flux is comparable to our $3 \sigma$ noise level, and hence a nondetection is unsurprising.

\subsection{Frequency of Massive Disks}

We detected 39 sources in our $1 \mathrm{~mm}$ mosaics (excluding the $\mathrm{BN}$ object). Of these, 32 correspond to (presumed) low-mass near-IR cluster members, and 6 (the "MM" sources) are detected only at $\gtrsim 1 \mathrm{~mm}$ wavelengths. The remaining detection, LMLA 162, while not listed as a near-IR source in Hillenbrand \& Carpenter (2000), is seen at $3.6 \mu \mathrm{m}$ (Lada et al. 2004); examination of an archival 2MASS image shows a weak $2 \mu \mathrm{m}$ source at this position as well. Since the millimeter-only detections are probably embedded, possibly spherical, protostellar objects ( $\S 4.1$ ), we exclude these from our discussion of disk statistics. Of the remaining 33 detections, $100 \%$ of the $1 \mathrm{~mm}$ emission can be attributed to hot gas (free-free) for 6 sources. Thus, we are left with 27 sources whose $1 \mathrm{~mm}$ emission (probably) traces dust in protoplanetary disks.

Since the noise varies across our images, these 27 sources are all detected above slightly different thresholds. To examine the frequency of disks more massive than some value, we make sensitivity cuts at various levels, examining only the statistics of sources detected above chosen noise levels.

We consider first the 115 cluster members surveyed to a $1 \sigma$ noise level of $2.7 \mathrm{mJy}$ or less. Sources detected above $3 \sigma$ have a circumstellar (dust + gas) mass of $\geq 0.01 M_{\odot}$. Nine sources $(8 \%)$ show dust emission of $\geq 8.1 \mathrm{mJy}$ (i.e., $3 \sigma$ detections at this noise level). If we use a higher noise cutoff of $5 \mathrm{mJy}$, then we find that 7 out of 193 stars exhibit dust emission above the $3 \sigma$ level of $15 \mathrm{mJy}$. So, $\sim 4 \%$ of stars have disks more massive than $0.02 M_{\odot}$. If we extend the sensitivity cutoff further, to $10 \mathrm{mJy}$, then $3 / 254$ or $\sim 1 \%$ of stars are seen with disks more massive than $0.04 M_{\odot}$. All of the $3 \sigma$ mass levels considered here fall within the range of estimates for the MMSN $\left(\sim 0.01-0.1 M_{\odot}\right.$; Weidenschilling 1977).

The percentage of high-mass disks derived here can be compared to that determined by Eisner \& Carpenter (2006). The observations presented here are substantially more sensitive than previous observations, and we probe the frequency of disks down to lower mass levels; we can therefore only compare statistics for the most massive disks in our sample. Eisner \& Carpenter (2006) found that $\leq 2 \%$ of cluster members in the ONC have disks more massive than $0.1 M_{\odot}$. They assumed a distance of $480 \mathrm{pc}$; their mass limit is actually only $0.07 M_{\odot}$ for the distance of $400 \mathrm{pc}$ assumed here. Here, we find that $\leqslant 1 \%$ of stars surveyed are surrounded by such massive disks, consistent with the estimate from Eisner \& Carpenter (2006).

We emphasize that results presented above (and in the following sections) depend on the conversion of $1.3 \mathrm{~mm}$ flux into mass. As discussed in $\S 3.4$, there may be some spread in the dust properties of our sample that could lead to variations in the derived circumstellar masses. For the roughly coeval, predominantly low-mass cluster population in the $\mathrm{ONC}$, we argued that this is a relatively small uncertainty.

\subsection{A Typical Disk in the ONC}

We computed the average flux for the ensemble of nondetected sources in $\S 3.6$. The average flux indicates that a "typical" nondetected source in the ONC likely possesses a disk with a mass of $0.0015 \pm 0.0003 M_{\odot}$. If we include detected objects (whose dust fluxes are listed in Table 2) in the ensemble, we find that the average disk mass for near-IR cluster members in the region is $\sim 0.0027 \pm 0.0002 M_{\odot}$.

This is comparable to the average mass determined for 23 proplyds in the ONC at $880 \mu \mathrm{m}$ (Williams et al. 2005), but substantially lower than the average mass determined for $>300$ stars 
at $3 \mathrm{~mm}$ wavelengths, $0.005 \pm 0.001 M_{\odot}$ (Eisner \& Carpenter 2006). The discrepancy can be explained in large part by contamination from free-free emission. Because the inferred dust mass is proportional to $\lambda^{3} S_{\nu}$ (eq. [3]), this contamination has a much greater effect on the masses inferred from the $3 \mathrm{~mm}$ data than on our estimates based on $1 \mathrm{~mm}$ data. The dust mass attributed to free-free emission is $(2.3)^{3} \approx 12$ times larger at $3 \mathrm{~mm}$ than at $1.3 \mathrm{~mm}$. If free-free emission is present at the $\lesssim 0.2 \mathrm{mJy}$ level ( 33.3 ), it would add $\lesssim 0.003 M_{\odot}$ to the average mass inferred at $3 \mathrm{~mm}$. Furthermore, if we recompute the mean disk mass from Eisner \& Carpenter (2006) using a distance of $400 \mathrm{pc}$, the average mass is decreased by $30 \%$. With the distance correction and the subtraction of potential free-free contamination, the recomputed average mass from Eisner \& Carpenter (2006) is $\gtrsim 0.001 M_{\odot}$, in agreement with the estimated average mass inferred from our $1 \mathrm{~mm}$ observations.

\subsection{Comparison of Disk and Exoplanet Frequencies}

Less than $10 \%$ of stars in the ONC possess disks comparable to the MMSN ( $\$ 4.2$ ). Moreover, the average mass measured for the ensemble of (individually) nondetected sources is 10 times smaller than even the low end of estimates for the MMSN ( $(4.3)$, indicating that the majority of stars do not possess enough mass to form Jupiter-mass planets.

These statistics can be compared with the frequency of Jupitermass planets found around nearby main-sequence stars. Of stars surveyed, $6 \%$ have a Jupiter-mass (or larger) planet within $5 \mathrm{AU}$, while an extrapolation based on current results suggests that up to $10 \%$ of stars could have a Jupiter-mass planet within $20 \mathrm{AU}$ (Marcy et al. 2005). The frequency of massive planets is comparable to the frequency of disks in the ONC with (low-end) minimum-mass solar nebulae. It appears that the MMSN, applied to disk-mass measurements like those presented here, is a reasonable criterion for forming massive, Jupiter-like planets in typical star-forming regions like Orion.

\subsection{Disk Evolution}

The frequency of massive disks in the ONC (aged $\sim 1 \mathrm{Myr}$ ) can be compared with surveys of rich clusters of different ages, NGC 2024 (aged $0.3 \mathrm{Myr}$ ) and IC 348 (aged 2 Myr), to constrain the evolution of disks in clustered star-forming environments. While this comparison has been made previously using $3 \mathrm{~mm}$ observations (e.g., Eisner \& Carpenter 2006), our $1 \mathrm{~mm}$ measurements are less contaminated by free-free emission and yield different results ( $\S 4.3)$. Although the surveys of NGC 2024 and IC 348 were at $3 \mathrm{~mm}$, the lack of $\mathrm{O}$ stars in those regions should produce less ionized gas, and hence less contamination by free-free emission than in the ONC.

The average disk masses for "typical" low-mass stars in the three regions are plotted as functions of cluster age in Figure 9. For the ONC, we infer a mean disk mass of $0.0027 \pm 0.0002$ (§ 4.3). In NGC 2024, the mean disk mass is $0.005 \pm 0.001 M_{\odot}$ (Eisner \& Carpenter 2003), compared to $0.002 \pm 0.001 M_{\odot}$ in IC 348 (Carpenter 2002). If the differences between NGC 2024, the ONC, and IC 348 are due to temporal evolution, these observations suggest that massive disks/envelopes dissipate on timescales $\lesssim 1 \mathrm{Myr}$, and that the average disk mass decreases by a factor of $1.9 \pm 0.4$ between $\sim 0.3$ and 1 Myr.

\subsection{Dependence of Disk Properties on Environment}

It has been suggested that circumstellar disks in clustered environments may be truncated due to close encounters with massive stars, resulting in either tidal stripping or photoevaporation of outer disk material (e.g., Scally \& Clarke 2001). Indeed, pho-

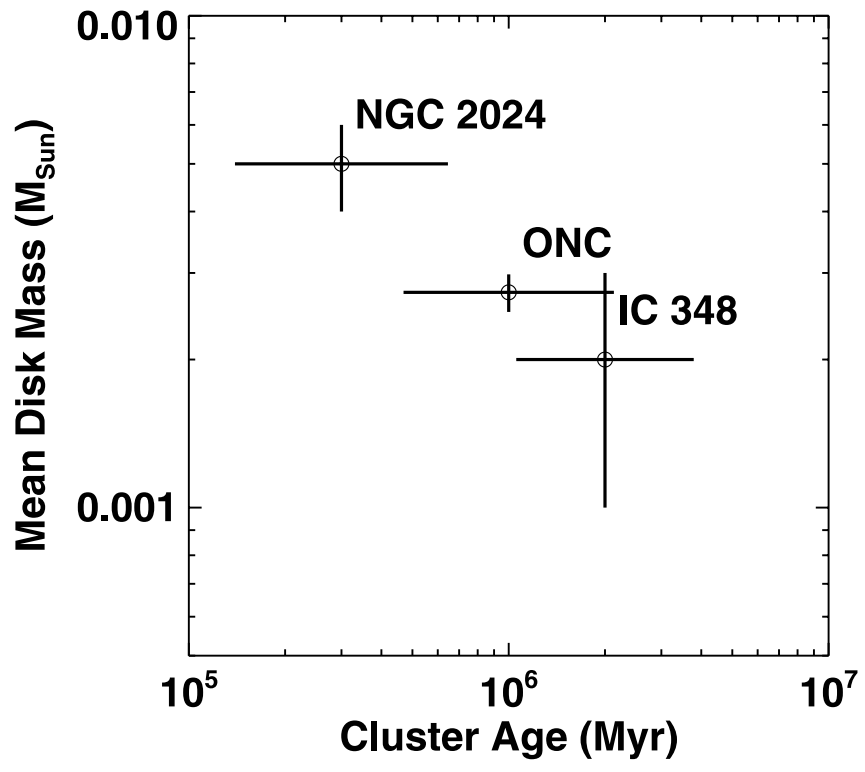

FIG. 9.- Average disk mass as a function of age for the NGC 2024, ONC, and IC 348 clusters. The disk masses are taken from this work, Eisner \& Carpenter (2003), and Carpenter (2002), and estimated cluster ages and uncertainties are from Meyer (1996), Ali (1996), Hillenbrand (1997), Luhman et al. (1998), and Luhman (1999). Average disk masses for NGC 2024 and IC 348 were measured at $3 \mathrm{~mm}$, where potential contributions from free-free emission would be stronger than for the average mass measured here for the $\mathrm{ONC}$ at $1 \mathrm{~mm}$. We argue in $\S 4.5$, however, that free-free contamination is unlikely in NGC 2024 and IC 348.

toevaporative mass loss has been observed from many proplyds, suggesting mass-loss rates as high as $10^{-7}-10^{-6} M_{\odot} \mathrm{yr}^{-1}$ (e.g., Henney \& O'Dell 1999), which would severely deplete the masses of disks over the $\sim 1$ Myr lifetime of the cluster. More detailed models have shown that the mass-loss rate should be substantially lower for disks with smaller outer radii, since disk material at smaller radii is more tightly gravitationally bound (Clarke 2007). A prediction of these models is that larger disks will also be the most massive, since they have to withstand higher photoevaporative mass-loss rates.

The proplyds detected in our observations are in the top $\sim 1 / 3$ of the size distribution inferred by Vicente \& Alves (2005). However, the emission from most of these is dominated by free-free emission, and even for objects where some component of the flux is due to dust, inferred masses are $\lesssim 0.01 M_{\odot}$. Furthermore, there are many other proplyds whose diameters are in the top $30 \%$ that are not detected in our observations. We also see no obvious trend of increasing flux with increasing angular size in our data (Table 2). Thus, we find little evidence that the most extended disks are the most massive.

Environmental effects on massive disks can also be investigated through the dependence of disk properties on cluster radius. We consider the positions of the disks detected in our observations (i.e., detected sources corresponding to known near-IR cluster member positions) relative to the cluster center, which we define to lie roughly in the middle of the four bright Trapezium stars at $(\alpha, \delta)_{\mathrm{J} 2000.0}=\left(5^{\mathrm{h}} 35^{\mathrm{m}} 16.34^{\mathrm{s}},-5^{\circ} 23^{\prime} 15.6^{\prime \prime}\right)$. Figure 10 shows that more massive disks tend to be found farther away from the Trapezium stars. If we consider only those 194 cluster members where we could have detected disks more massive than $0.02 M_{\odot}$ above the $3 \sigma$ noise level, we find disks around 1/84 stars $(\sim 1 \%)$ within $30^{\prime \prime}$ and $6 / 110$ stars $(\sim 5 \%)$ at radii larger than $30^{\prime \prime}$. Fisher's exact test indicates an $86 \%$ probability $(1.5 \sigma)$ that the small and large cluster radii sources have different frequencies of massive disks. 


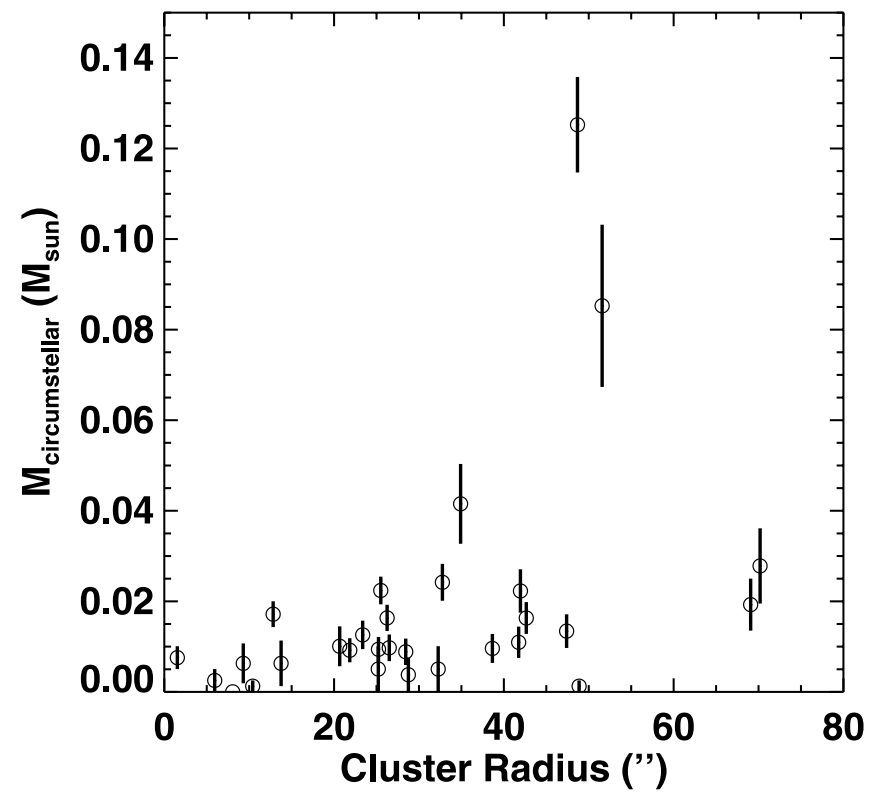

FIG. 10.-Circumstellar mass as a function of radial distance from the center of the Trapezium stars for detected objects.

Finally, comparison of the ONC with the lower stellar density Taurus region provides another test of whether the massive $\mathrm{O}$ stars and high stellar density in the Trapezium region lead to different disk properties than in more "benign" environments. As discussed in $\S 4.2$, we detected disks more massive than $0.01 M_{\odot}$ around $9 / 115(\sim 8 \%)$ low-mass ONC cluster members. For comparison, 34/153 ( 22\%) of Taurus stars possess such massive disks (Andrews \& Williams 2005). Fisher's exact test yields a $>99 \%$ probability $(3 \sigma)$ that the frequencies of $0.01 M_{\odot}$ disks in Taurus and the ONC are different. For a slightly higher mass cutoff of $0.04 M_{\odot}$, such massive disks are found around $<1 \%$ of stars in the ONC, compared to $\sim 5 \%$ for Taurus. These percentages indicate a $>99 \%$ probability that the underlying distribution of $0.04 M_{\odot}$ disks in Taurus and the ONC differ. The fraction of approximately MMSN-mass disks in Orion is substantially smaller than in Taurus, substantiating the hypothesis that the rich cluster environment may play a role in limiting the number of massive disks.

This conclusion differs from that of Eisner \& Carpenter (2006), where the statistics of disks more massive than $0.1 M_{\odot}$ were found to be statistically indistinguishable in Taurus and the ONC. Using the revised distance of $400 \mathrm{pc}$ changes the conclusion of Eisner $\&$ Carpenter (2006) because if statistics of (distance-corrected) $0.07 M_{\odot}$ disks are compared, they are found to be substantially more common in Taurus (Fisher's exact test indicates only an $\sim 1.3 \%$ probability that the two distributions are the same). Furthermore, the $1 \mathrm{~mm}$ observations presented here are sensitive to much more of the disk-mass distribution, allowing a more robust comparison between Taurus and the ONC.

\subsection{Correlation of Circumstellar and Stellar Masses}

Several spectroscopic surveys have provided accurate masses for a subset of the stellar population encompassed by our mosaics (Hillenbrand 1997; Luhman et al. 2000; Slesnick et al. 2004). By examining these surveys, after registration of the positions of detected sources to the 2 MASS grid, we find $\sim 130$ objects with spectroscopically determined masses within the unit-gain contour of our mosaic. While the stellar masses of the remaining cluster

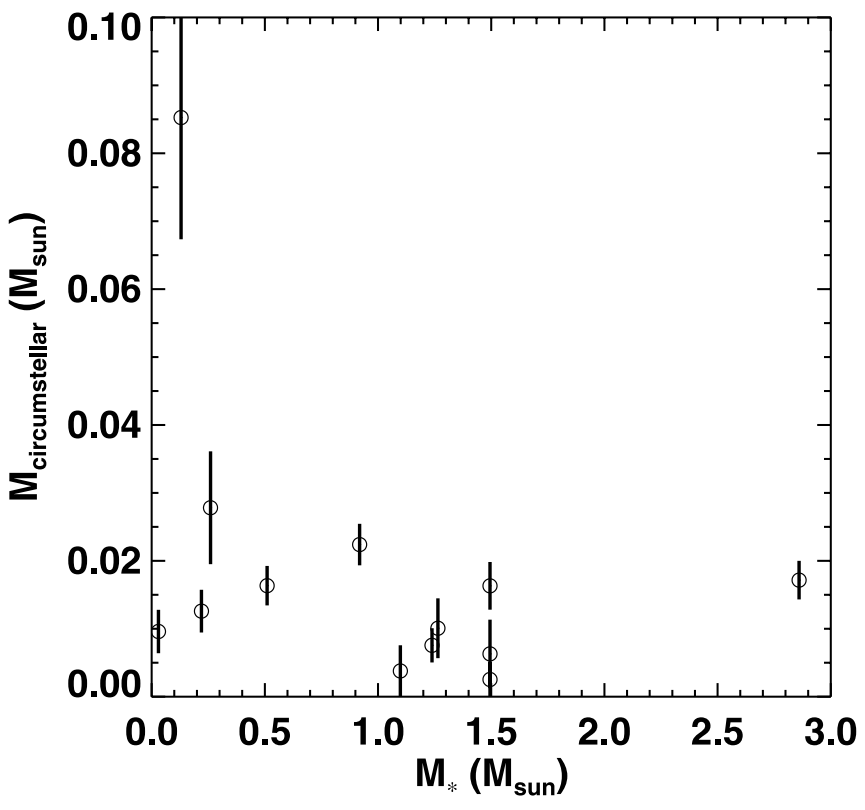

FIG. 11.-Circumstellar mass as a function of stellar mass for the subset of detected objects where spectroscopically determined stellar masses are available (Hillenbrand 1997; Luhman et al. 2000; Slesnick et al. 2004).

members contained in our mosaic have been estimated statistically by dereddening stars so that they fall on the expected isochrone for the ONC (Hillenbrand \& Carpenter 2000), the masses of individual stars determined in this way have large uncertainties, and we do not use them here.

To examine how disk mass depends on stellar mass, we divide the $\sim 130$ sources with spectroscopically determined masses into three mass bins containing roughly equal numbers of stars. The first bin contains stars less massive than $0.3 M_{\odot}$, the second bin includes stars with masses between 0.3 and $1.0 M_{\odot}$, and the third bin contains stars with masses between 1.0 and $10.0 M_{\odot}$. We then make a further cut by excluding all objects for which the noise in our $1 \mathrm{~mm}$ mosaic at the source position is greater than some cutoff value. As in $\S 4.2$, we consider a noise cutoff of $2.7 \mathrm{mJy}$, which provides a corresponding $3 \sigma$ circumstellar-mass threshold of $0.01 M_{\odot}$. Ideally, we would also bin this sample by cluster radius to control for potential mass segregation in the inner regions of the ONC (e.g., Hillenbrand 1997). Unfortunately, we lack a sufficiently large sample to do this here.

If we use the raw image fluxes (Table 1), we find more sources detected in the highest stellar-mass bin. For 65 stars with spectroscopically determined masses, surveyed with a noise level of 2.7 mJy or lower, we detect $1 / 21(5 \%)$ stars with $M_{*}<0.3 M_{\odot}$, $2 / 24(8 \%)$ stars with $0.3 M_{\odot}<M_{*}<1 M_{\odot}$, and $6 / 20(30 \%)$ stars with $M_{*}>1 M_{\odot}$. However, the higher percentage of detected sources in the highest stellar-mass bin is due entirely to contamination by free-free emission: if we use dust fluxes from Table 2, then $0 / 20$ stars in the highest stellar-mass bin are detected. We infer, therefore, that higher mass stars are more likely to exhibit free-free emission.

In contrast, it seems that more massive stars may be less likely to possess massive circumstellar disks. Using the dust fluxes from Table 2, we find that out of the 65 stars discussed above, we detect dust emission above the $3 \sigma$ level toward $1 / 21(5 \%)$ stars with $M_{*}<0.3 M_{\odot}, 2 / 24(8 \%)$ stars with $0.3 M_{\odot}<M_{*}<$ $1 M_{\odot}$, and $0 / 20(0 \%)$ stars with $M_{*}>1 M_{\odot}$. This suggests a lower frequency of disks around stars more massive than $1 M_{\odot}$, 
but these small-number statistics do not allow a definite conclusion. A plot of inferred circumstellar disk masses versus stellar masses (where available) supports the hypothesis that the most massive disks are found around the lowest mass stars. This trend is not, however, statistically significant for the small number of objects in Figure 11. A similar picture was seen in Taurus (Andrews \& Williams 2005), but no correlation between stellar mass and disk mass could be established, although the most massive disks were found around stars less massive than $1 M_{\odot}$. We note that Natta et al. (2000) claimed to see a correlation between disk and stellar masses around early-type stars; however, the dispersion is large, and the significance of the trend is marginal.

\section{CONCLUSIONS}

We imaged a $2^{\prime} \times 2^{\prime}$ region of the Orion Nebula Cluster in $1.3 \mathrm{~mm}$ wavelength continuum emission with CARMA and the SMA. Out of $>250$ known near-IR cluster members, we detected $1.3 \mathrm{~mm}$ emission above the $3 \sigma$ noise level toward 33. In addition, we detected $1 \mathrm{~mm}$ emission above the $5 \sigma$ noise level from six sources not associated with shorter wavelength counterparts. Several of these detected objects are spatially resolved with our observations, indicating sizes of $\sim 250-450 \mathrm{AU}$.

Modeling of long-wavelength fluxes for our targets allowed separation of dust and free-free emission components in the measured fluxes. We showed that for the majority of detected sources, the $1 \mathrm{~mm}$ emission appears to trace warm, optically thin, circumstellar dust. However, for many of the proplyds, which are located close to the Trapezium stars, the millimeter-wavelength emission is dominated by thermal free-free emission from hot, ionized gas.

Dust masses inferred for detected sources range from 0.01 to $0.5 M_{\odot}$. For the $\sim 225$ known near-IR cluster members not detected in our $1 \mathrm{~mm}$ observations, images toward the positions of near-IR sources were stacked to constrain the mean flux, and circumstellar mass, of the ensemble. The average flux is detected at the $>4 \sigma$ confidence level and implies an average disk mass of $\sim 0.001 M_{\odot}$, approximately an order of magnitude smaller than the minimum-mass solar nebula. Even when detected sources are included, the average mass is $<0.003 M_{\odot}$. While the derived masses are uncertain by a factor of 3 or so (mostly due to uncertainties in the dust opacity), the range of possible average disk masses is still smaller than even the low end of estimates for the MMSN. A "typical" star in the ONC does not appear to possess sufficient mass in small dust grains to form Jupiter-mass (or larger) planets. Evidently, giant-planet formation is either advanced (having thus depleted the small dust grains in the disk) or impossible around most stars in the ONC.
We compared the average disk mass inferred for the ONC with similarly determined average masses in older and younger clusters. We find evidence for evolution of the dust (most likely depletion or agglomeration) on $\sim 1 \mathrm{Myr}$ timescales. Between $\sim 0.3$ and $\sim 1$ Myr, the average disk mass decreases by a factor of $1.9 \pm 0.4$.

The percentage of stars in Orion surrounded by disks more massive than the minimum-mass solar nebula is $<10 \%$. This is significantly lower than in Taurus, indicating that environment has an impact on the disk-mass distribution. Our data suggest (with marginal statistical significance) that the most massive disks may be located further from the Trapezium stars, supporting the hypothesis that photoevaporation may be truncating disks near to the cluster center.

Finally, our observations show no clear correlation between stellar mass and disk mass, but suggest that massive disks may be more likely to be found around lower mass stars. The percentage of detected disks is lower for stars more massive than $1 M_{\odot}$, and the most massive disks detected are associated with the relatively low-stellar-mass stars in the sample. However, larger numbers of stellar- and disk-mass measurements in the ONC are needed to build up better statistics and further constrain the relationship between stellar and disk properties.

J. A. E. acknowledges support from a Miller Research Fellowship. The authors thank M. Wright for his assistance in simulating CARMA mosaics in order to choose the optimal mosaic spacings for the observations. This publication makes use of data products from the Two Micron All Sky Survey (2MASS), which is a joint project of the University of Massachusetts and the Infrared Processing and Analysis Center (IPAC), funded by NASA and the National Science Foundation. 2MASS science data and information services were provided by the Infrared Science Archive at IPAC. Support for CARMA construction was derived from the states of Illinois, California, and Maryland, the Gordon and Betty Moore Foundation, the Kenneth T. and Eileen L. Norris Foundation, the Associates of the California Institute of Technology, and the National Science Foundation. Ongoing CARMA development and operations are supported by the National Science Foundation under a cooperative agreement and by the CARMA partner universities. The Submillimeter Array is a joint project between the Smithsonian Astrophysical Observatory and the Academia Sinica Institute of Astronomy and Astrophysics, and is funded by the Smithsonian Institution and the Academia Sinica.

\section{APPENDIX}

\section{FREE-FREE EMISSION SPECTRUM FOR AN IONIZED WIND}

We compute the spectrum expected for free-free emission from ionized gas whose density depends on stellocentric radius as

$$
n_{\text {gas }}=n_{0}\left(R / R_{0}\right)^{-\alpha}
$$

For an ionized wind with constant $\dot{M}$, such as we expect for proplyds, $\alpha=2$. However, massive stars may ionize their circumstellar environments directly, in which case the density distribution may differ from this $R^{-2}$ power law.

For a power-law density profile (eq. [A1]), the optical depth can be approximated as (e.g., Altenhoff et al. 1960)

$$
\tau_{\nu, \mathrm{ff}} \approx \int_{0}^{\infty} \frac{0.16 n_{0}^{2}}{\nu^{2.1} T_{e}^{1.35}} \frac{d z}{\left(x^{2}+z^{2}\right)^{\alpha}}
$$


Here, $z$ is the line of sight through the ionized gas in pc, $x$ is the impact parameter in $\mathrm{pc}, n_{0}$ is the normalization of the gas density in $\mathrm{cm}^{-3}$ (assumed to represent the electron and ion densities), $T_{e}$ is the electron temperature, and $\nu$ is the frequency in GHz. This expression can be integrated directly as long as $\alpha \geq 1.5$, with the result

$$
\tau_{\nu, \mathrm{ff}} \approx \frac{0.08 n_{0}^{2} R_{0}^{4}}{\nu^{2.1} T_{e}^{1.35}} x^{-(2 \alpha-1)} \frac{\pi(-1)^{\alpha-1} \Gamma(1 / 2)}{\sin (\pi / 2)(\alpha-1) ! \Gamma[1 /(2-\alpha+1)]} .
$$

The last fraction has order unity, and we can thus approximate $\tau$ as

$$
\tau_{\nu, \mathrm{ff}} \approx \frac{0.08 n_{0}^{2} R_{0}^{4}}{\nu^{2.1} T_{e}^{1.35}} x^{-(2 \alpha-1)} .
$$

We can now invert equation (A4) to determine the maximum impact parameter for which $\tau \gtrsim 1$,

$$
x_{\tau \approx 1} \approx\left(\frac{0.08 n_{0}^{2} R_{0}^{4}}{\nu^{2.1} T_{e}^{1.35}}\right)^{1 / 2 \alpha-1}
$$

For impact parameters larger than $x_{\tau \approx 1}$, the gas is optically thin, and at smaller impact parameters the gas is optically thick. The total spectrum of free-free emission from the source can be approximated by the blackbody flux times the solid angle of the optically thick region, as long as the optically thick region is finite. When the entire wind becomes optically thin, the spectrum flattens.

We can thus parameterize the free-free emission from a wind as

$$
F_{\nu, \text { ff }}= \begin{cases}F_{\nu, \text { turn }}\left(\nu / \nu_{\text {turn }}\right)^{-0.1} & \nu \geq \nu_{\text {turn }} \\ F_{\nu, \text { turn }}\left(\nu / \nu_{\text {turn }}\right)^{(4 \alpha-6.2) /(2 \alpha-1)} & \nu \leq \nu_{\text {turn }}\end{cases}
$$

(see Wright \& Barlow 1975 for a somewhat different derivation of this result). For a spherical wind with constant $\dot{M}, \alpha=2$ and the optically thick material emits with $F_{\nu} \propto \nu^{0.6}$. Steeper exponents can result if the ionized gas density drops off more steeply than $R^{-2}$, for example, as might occur in a centrally illuminated wind from a massive star such as BN (e.g., Plambeck et al. 1995). Alternatively, nonspherical wind geometries can lead to shallower radial density profiles, and hence shallower spectral slopes of the free-free emission (Wright \& Barlow 1975). For simplicity, since we generally have a limited number of data points with which to constrain the free-free emission spectrum, we will assume that $\alpha=2$ in the present analysis.

\section{REFERENCES}

Ali, B. 1996, Ph.D. thesis, The Ohio State University

Alibert, Y., Mordasini, C., Benz, W., \& Winisdoerffer, C. 2005, A\&A, 434, 343

Altenhoff, W. J., Mezger, P. G., Wendker, H., \& Westerhout, G. 1960, Veröff.

Sternw. Bonn, 59, 48

André, P., \& Montmerle, T. 1994, ApJ, 420, 837

Andrews, S. M., \& Williams, J. P. 2005, ApJ, 631, 1134 2007, ApJ, 671, 1800

Bachiller, R. 1996, ARA\&A, 34, 111

Bally, J., O’Dell, C. R., \& McCaughrean, M. J. 2000, AJ, 119, 2919

Bally, J., Sutherland, R. S., Devine, D., \& Johnstone, D. 1998a, AJ, 116, 293

Bally, J., Testi, L., Sargent, A., \& Carlstrom, J. 1998b, AJ, 116, 854

Beckwith, S. V. W., Sargent, A. I., Chini, R. S., \& Guesten, R. 1990, AJ, 99, 924

Bower, G. C., Plambeck, R. L., Bolatto, A., McCrady, N., Graham, J. R., de Pater, I., Liu, M. C., \& Baganoff, F. K. 2003, ApJ, 598, 1140

Carpenter, J. M. 2000, AJ, 120, 3139

- 2002, AJ, 124, 1593

Clarke, C. J. 2007, MNRAS, 376, 1350

D’Antona, F., \& Mazzitelli, I. 1994, ApJS, 90, 467

Dutrey, A., Guilloteau, S., Duvert, G., Prato, L., Simon, M., Schuster, K., \& Menard, F. 1996, A\&A, 309, 493

Eisner, J. A., \& Carpenter, J. M. 2003, ApJ, 598, 1341 2006, ApJ, 641, 1162

Eisner, J. A., Lane, B. F., Hillenbrand, L., Akeson, R., \& Sargent, A. 2004, ApJ, 613, 1049

Felli, M., Churchwell, E., Wilson, T. L., \& Taylor, G. B. 1993a, A\&AS, 98, 137

Felli, M., Taylor, G. B., Catarzi, M., Churchwell, E., \& Kurtz, S. 1993b, A\&AS, 101,127

Forbrich, J., Menten, K. M., \& Reid, M. J. 2008, A\&A, 477, 267

Furuya, R. S., Shinnaga, H., Nakanishi, K., Momose, M., \& Saito, M. 2003, PASJ, 55, L83

Garay, G., Moran, J. M., \& Reid, M. J. 1987, ApJ, 314, 535

Genzel, R., Reid, M. J., Moran, J. M., \& Downes, D. 1981, ApJ, 244, 884

Gezari, D. Y., Backman, D. E., \& Werner, M. W. 1998, ApJ, 509, 283

Haisch, K. E., Lada, E. A., \& Lada, C. J. 2001, AJ, 121, 2065
Hayashi, C. 1981, Prog. Theor. Phys. Suppl., 70, 35

Henney, W. J., \& O'Dell, C. R. 1999, AJ, 118, 2350

Henning, T., Pfau, W., Zinnecker, H., \& Prusti, T. 1993, A\&A, 276, 129

Hester, J. J., \& Desch, S. J. 2005, in ASP Conf. Ser. 341, Chondrites and the Protoplanetary Disk, ed. A. N. Krot, E. R. D. Scott, \& B. Reipurth (San Francisco: ASP), 107

Hildebrand, R. H. 1983, QJRAS, 24, 267

Hillenbrand, L. A. 1997, AJ, 113, 1733

Hillenbrand, L. A., \& Carpenter, J. M. 2000, ApJ, 540, 236

Hillenbrand, L. A., Strom, S. E., Calvet, N., Merrill, K. M., Gatley, I., Makidon, R. B., Meyer, M. R., \& Skrutskie, M. F. 1998, AJ, 116, 1816

Hogerheijde, M. R., Jayawardhana, R., Johnstone, D., Blake, G. A., \& Kessler, J. E. $2002, \mathrm{AJ}, 124,3387$

Itoh, Y., et al. 2003, ApJ, 586, L141

Koerner, D. W., \& Sargent, A. I. 1995, AJ, 109, 2138

Kraus, S., et al. 2007, A\&A, 466, 649

Lada, C. J., Depoy, D. L., Merrill, K. M., \& Gatley, I. 1991, ApJ, 374, 533

Lada, C. J., \& Lada, E. A. 2003, ARA\&A, 41, 57

Lada, C. J., Muench, A. A., Haisch, K. E., Lada, E. A., Alves, J. F., Tollestrup, E. V., \& Willner, S. P. 2000, AJ, 120, 3162

Lada, C. J., Muench, A. A., Lada, E. A., \& Alves, J. F. 2004, AJ, 128, 1254

Lada, E. A., Strom, K. M., \& Myers, P. C. 1993, in Protostars and Planets III, ed. E. H. Levy \& J. I. Lunine (Tuscon: Univ. Arizona Press), 245

Luhman, K. L. 1999, ApJ, 525, 466

Luhman, K. L., Rieke, G. H., Lada, C. J., \& Lada, E. A. 1998, ApJ, 508, 347

Luhman, K. L., Rieke, G. H., Young, E. T., Cotera, A. S., Chen, H., Rieke, M. J., Schneider, G., \& Thompson, R. I. 2000, ApJ, 540, 1016

Marcy, G., Butler, R. P., Fischer, D., Vogt, S., Wright, J. T., Tinney, C. G., \& Jones, H. R. A. 2005, Prog. Theor. Phys. Suppl., 158, 24

McCaughrean, M. J., \& O’Dell, C. R. 1996, AJ, 111, 1977

Menten, K. M., Reid, M. J., Forbrich, J., \& Brunthaler, A. 2007, A\&A, 474, 515

Meyer, M. R. 1996, Ph.D. thesis, Univ. Massachusetts

Motte, F., \& André, P. 2001, A\&A, 365, 440

Motte, F., André, P., \& Neri, R. 1998, A\&A, 336, 150 
Mundy, L. G., Looney, L. W., \& Lada, E. A. 1995, ApJ, 452, L137

Natta, A., Grinin, V., \& Mannings, V. 2000, Protostars and Planets IV, ed. V. Mannings, A. P. Boss, \& S. S. Russell (Tucson: Univ. Arizona Press), 559

Nuernberger, D., Brandner, W., Yorke, H. W., \& Zinnecker, H. 1998, A\&A, 330,549

Nuernberger, D., Chini, R., \& Zinnecker, H. 1997, A\&A, 324, 1036

O’Dell, C. R., Wen, Z., \& Hu, X. 1993, ApJ, 410, 696

O’Dell, C. R., \& Wong, K. 1996, AJ, 111, 846

Osterloh, M., \& Beckwith, S. V. W. 1995, ApJ, 439, 288

Padgett, D. L., Brandner, W., Stapelfeldt, K. R., Strom, S. E., Terebey, S., \& Koerner, D. 1999, AJ, 117, 1490

Plambeck, R. L., Wright, M. C. H., Mundy, L. G., \& Looney, L. W. 1995, ApJ, 455, L189

Pollack, J. B., Hollenbach, D., Beckwith, S., Simonelli, D. P., Roush, T., \& Fong, W. 1994, ApJ, 421, 615

Prosser, C. F., Stauffer, J. R., Hartmann, L., Soderblom, D. R., Jones, B. F., Werner, M. W., \& McCaughrean, M. J. 1994, ApJ, 421, 517

Sandstrom, K. M., Peek, J. E. G., Bower, G. C., Bolatto, A. D., \& Plambeck, R. L. 2007, ApJ, 667, 1161

Sault, R. J., Teuben, P. J., \& Wright, M. C. H. 1995, in ASP Conf. Ser. 77, Astronomical Data Analysis Software and Systems IV (San Francisco: ASP), 433

Scally, A., \& Clarke, C. 2001, MNRAS, 325, 449
Slesnick, C. L., Hillenbrand, L. A., \& Carpenter, J. M. 2004, ApJ, 610, 1045 Smith, N., Bally, J., Shuping, R. Y., Morris, M., \& Kassis, M. 2005, AJ, 130, 1763

Strom, K. M., Strom, S. E., Edwards, S., Cabrit, S., \& Skrutskie, M. F. 1989, AJ, 97, 1451

Testi, L., \& Sargent, A. I. 1998, ApJ, 508, L91

Vicente, S. M., \& Alves, J. 2005, A\&A, 441, 195

Weidenschilling, S. J. 1977, Ap\&SS, 51, 153

Williams, J. P., Andrews, S. M., \& Wilner, D. J. 2005, ApJ, 634, 495

Williams, J. P., \& Gaidos, E. 2007, ApJ, 663, L33

Wilner, D. J., Ho, P. T. P., Kastner, J. H., \& Rodríguez, L. F. 2000, ApJ, 534, L101

Wright, A. E., \& Barlow, M. J. 1975, MNRAS, 170, 41

Zapata, L. A., Ho, P. T. P., Rodríguez, L. F., O'Dell, C. R., Zhang, Q., \& Muench, A. 2006, ApJ, 653, 398

Zapata, L. A., Ho, P. T. P., Rodríguez, L. F., Schilke, P., \& Kurtz, S. E. 2007, A\&A, 471, L59

Zapata, L. A., Rodríguez, L. F., Ho, P. T. P., Zhang, Q., Qi, C., \& Kurtz, S. E. 2005, ApJ, 630, L85

Zapata, L. A., Rodríguez, L. F., Kurtz, S. E., O’Dell, C. R., \& Ho, P. T. P. 2004, ApJ, 610, L121

Ziurys, L. M., Wilson, T. L., \& Mauersberger, R. 1990, ApJ, 356, L25 